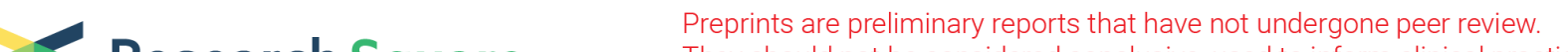 $\begin{array}{ll}\text { Research Square } & \begin{array}{l}\text { They should not be considered conclusive, used to inform clinical practice, } \\ \text { or referenced by the media as validated information. }\end{array}\end{array}$
}

\section{A Unilateral Robotic Knee Exoskeleton to Assess the Role of Natural Gait Assistance in Hemiparetic Patients}

Julio Salvador Lora-Millan ( $\sim$ julio.lora@csic.es )

Spanish National Research Council https://orcid.org/0000-0001-5968-5786

Francisco José Sanchez-Cuesta

Francisco de Vitoria University

Juan Pablo Romero

Universidad Francisco de Vitoria

Juan C. Moreno

Cajal Insititute. Spanish National Research Council.

\section{Eduardo Rocon}

Centro de Automática y Robótica. Consejo Superior de Investigaciones Científicas - Universidad Politécnica de Madrid

\section{Research}

Keywords: Hemiparetic gait, Stroke patients, Robotic exoskeleton, Unilateral assistance, Gait symmetry

Posted Date: November 18th, 2021

DOI: https://doi.org/10.21203/rs.3.rs-1070568/v1

License: (c) (1) This work is licensed under a Creative Commons Attribution 4.0 International License. Read Full License 


\section{A Unilateral Robotic Knee Exoskeleton to Assess \\ 2 the Role of Natural Gait Assistance in Hemiparetic \\ 3 Patients}

4 Julio Salvador Lora-Millán ${ }^{1 *}$, Francisco José Sanchez-Cuesta ${ }^{2}$, Juan Pablo Romero ${ }^{2,3}$, Juan C.

5 Moreno $^{4}$, Eduardo Rocon ${ }^{1}$

$6{ }^{1}$ Centro de Automática y Robótica, Consejo Superior de Investigaciones Científicas -

$7 \quad$ Universidad Politécnica de Madrid, Madrid, Spain

$8 \quad{ }^{2}$ Facultad de Ciencias Experimentales, Universidad Francisco de Vitoria, Pozuelo de Alarcón,

$9 \quad$ Madrid, Spain

$10 \quad{ }^{3}$ Brain Damage Unit, Hospital Beata María Ana, Madrid, Spain

$11{ }^{4}$ Neural Rehabilitation Group, Cajal Institute, Spanish National Research Council (CSIC),

12 Madrid, Spain

13 * Corresponding Author: julio.lora@csic.es

\section{Abstract}

15 Background: Hemiparetic gait is characterized by strong asymmetries that could 16 severely affect the quality of life of stroke survivors. This asymmetry is due to motor

17 deficits in the paretic leg and the resulting compensations in the non-paretic limb. In this

18 study, we aim to evaluate the effect of actively promoting gait symmetry in hemiparetic

19 patients by assessing the behavior of both paretic and non-paretic lower limbs. This

20 paper introduces the design and validation of the REFLEX prototype, a unilateral active

21 Knee-Ankle-Foot Orthosis able to naturally assist the paretic limb of hemiparetic

22 patients during gait. 
Methods: REFLEX uses an Adaptive Frequency Oscillator to estimate the continuous gait phase of the non-paretic limb. Based on this estimation, the device synchronically assists the paretic leg following two different control strategies: (1) Replicating the movement of the sound leg or (2) Inducing a healthy gait pattern on the paretic leg. Technical validation of the system was implemented on three healthy subjects, while the effect of the generated assistance was assessed in three stroke patients.

Results: Preliminary results proved the feasibility of the REFLEX prototype to assist gait by reinforcing symmetry. They also pointed out that the assistance of the paretic leg resulted in a decrease of the compensatory strategies developed by the non-paretic limb to achieve a functional gait. Notably, better results were attained when the assistance was provided according to a standard healthy pattern, which initially might suppose a lower symmetry but enabled a healthier evolution of the motion of the non-paretic limb.

Conclusions: This work presents the preliminary validation of the REFLEX prototype, a unilateral knee exoskeleton for gait assistance in hemiparetic patients. The compensatory mechanisms developed by their sound limb.

Keywords: Hemiparetic gait, Stroke patients, Robotic exoskeleton, Unilateral assistance, Gait symmetry

\section{Background}

causes of long-term disability worldwide [1,2]. Due to population aging and improved survival rates, it is estimated that the number of people living with stroke increases by $27 \%$ between 
$47[4,5]$, and $65 \%$ presents gait impairment [6] that affects their independence and quality of life

$48 \quad[7,8]$ and hampers the performance of daily life activities [9].

Hemiparetic gait is the most common gait disturbance due to stroke, and it is characterized by a strong asymmetric gait pattern due to contralateral motor weakness, motor control deficits, sensory and/or proprioceptive loss, and/or ataxia [10]. This asymmetrical gait supposes poor single limb support and uncontrolled forward movement [11]. Consequently, adaptive processes in the non-paretic limb occur to compensate for this misfunction [12].

For example, the swing phase in the non-paretic leg is shortened, and the stance phase is lengthened compared with the paretic limb, which leads to temporal asymmetry [9]. Regarding step length asymmetry, it is highly variable in stroke patients and representative of different compensatory strategies, and in patients with symmetric step length, it does not necessarily imply symmetric forward propulsion [13]. Other typical compensation mechanisms are pelvic hike or circumduction to overcome reduced foot clearance during paretic swing [12].

Despite the physical therapy, that can improve speed and endurance of hemiparetic subjects, asymmetric gait can be resistant to intervention [14] and is still present in 50\% of community-dwelling chronic stroke patients [15]. Hemiparetic gait supposes several consequences such as musculoskeletal pathologies in the non-paretic limb, falls due to instability, slow gait velocity, or increased energy consumption $[15,16]$. It is also an important factor for people with stroke, who are concerned about walking appearance [13,17].

In this context where motor recovery after stroke remains a clinical challenge [18], robotic exoskeletons have been presented, not only as rehabilitation tools [19], but also as assistive devices for hemiparetic subjects. Several authors have developed robotic devices to improve post-stroke gait quality, and some of them opted for bilateral configurations to assist and/or measure both paretic and non-paretic legs, such as the HAL exoskeleton [20,21], or the Curara prototype [22]. 
that only the paretic leg wore the device. These devices present several advantages as they are simpler and lighter than bilateral devices [23], and they can target the specific function of the affected joint during gait [24]. However, this configuration needs to address the appropriate coordination between the movements of the non-assisted joints and the assisted ones. For example, the Tibion robotic knee orthosis [25] or the Soft Ankle Exosuit from Harvard University [26,27] based their action on detecting gait events and reported improvements in the gait symmetry of stroke patients. ALEX II exoskeleton also improved the gait symmetry in post-stroke subjects [28], although its action followed a force field around a healthy foot trajectory exploiting the inter-joint coupling to coordinate the assistance.

Although the studies mentioned above have demonstrated the feasibility of robotic assistance to hemiparetic gait, several aspects have not yet been fully addressed, such as the users' adaptation to this assistance or the differences between assistive strategies. In this article, we present the robotic exoskeleton REFLEX (symmetRy rEinForcer uniLateral powEred eXoskeleton) as a tool to investigate these adaptation processes and the effect of unilateral gait assistance on hemiparetic subjects. This device was designed to assist the motion of the paretic knee of stroke survivors to promote a symmetric gait by guiding its movement during the swing phase and reinforcing the knee joint during the stance phase of the gait, as it was previously reported a successful target [25,29]. In this framework, we defined two different strategies to control the robotic exoskeleton: (1) to replicate the movement of the non-paretic limb or (2) to synchronically apply a healthy gait pattern to guide the movement of the limb.

The general objective of developing these strategies is to enable the intuitive control of the device that improves the embodiment of the technology by enabling a natural interaction between the user and the robotic exoskeleton. We hypothesize that the proper and natural interaction between these two systems will enable the patients to consider the robot's action as a part of their own gait capability, improving their gait quality as a consequence. Hemiparetic asymmetry is not only due to impairments in the affected limb, but it is also the consequence of 
100 prototype thus serves as a tool to assess the adaptation process of the subject to the exoskeleton 101 assistance and to evaluate the effects of such human-robot interaction in both paretic and non102 paretic legs.

\section{Methods}

\section{Robotic Exoskeleton Design}

The REFLEX prototype is a Knee-Ankle-Foot orthosis (KAFO) composed of two joints aligned to the knee and ankle of the user (Figure 1). The segments' length and the braces' positions can be tailored to the anthropometry of different users. The material of the major part of the prototype is Aluminum 7075, so the result is a resistant and light-weight device.

The knee joint is actuated by a DC brushless motor EC-60 flat 408057 (Maxon ag,

110 Switzerland) coupled with a harmonic drive CSD-20-160-2AGR (Harmonic Drive LLC,

111 EE.UU.). The transmission ratio of 1:60 of this system enables the application of a mean torque

112 of $35 \mathrm{Nm}$, required to perform the limb's movement [30]. The ankle joint of the prototype 113 remains non-actuated and unlimited, enabling its free movement in the sagittal plane. The total

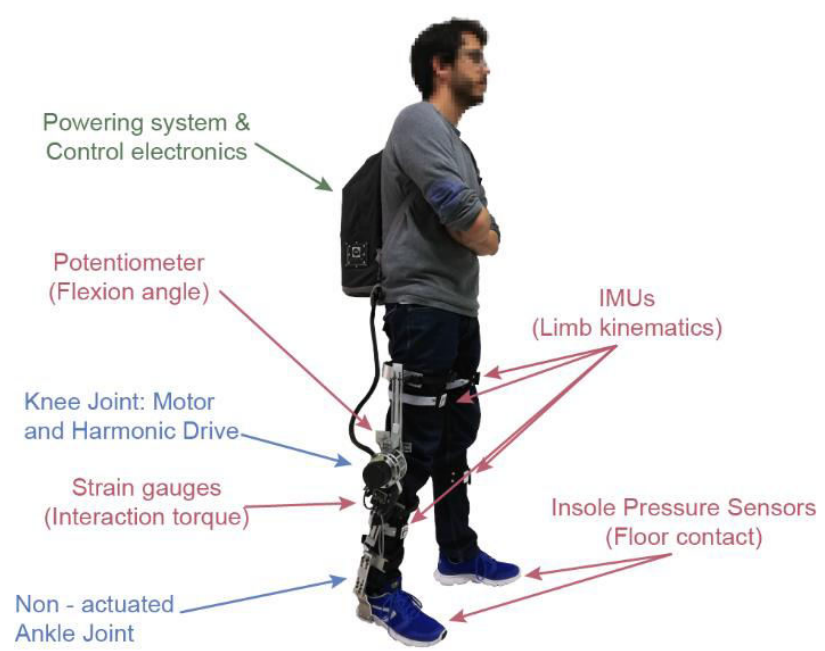

Figure 1: REFLEX prototype for the assistance of the knee joint of the paretic leg. This joint is actuated by a DC motor coupled to a Harmonic Drive, while the ankle remains unactuated. The sensors of the prototype are a potentiometer to measure the exoskeleton flexion in the sagittal plane, strain gauges to measure the interaction torque, inertial sensors (IMUs) to acquire the limbs kinematics, and insole pressure sensors to detect floor contact events. 
weight of the $\mathrm{KAFO}$ is about $4 \mathrm{~kg}$. The prototype is equipped with four sets of sensors that provide information on system variables that are used for the control in real-time:

1. A potentiometer, coupled with the joint axis, to measure the flexion/extension angle of the active joint. This information enables the robot to follow trajectories using a position closed-loop control algorithm.

2. An interaction torque sensor placed between the robot and the user. It consists of two pairs of strain gauges in a full Wheatstone bridge. This interaction torque is used to implement an impedance controller that adjusts the torque provided by the robotic system to the user's leg. This controller is fully described in section 2.1.3.

3. Three insole pressure sensors based on FSRs (Force Sensing Resistors) to assess the contact of each user's foot with the floor. These measurements distinguish between swing and stance phases and adapt the controllers accordingly.

4. Four Inertial Measurement Units (IMUs) (TechMCS, Technaid, Spain) to compute the kinematics of both legs. These sensors are attached to the shanks and thighs of the two legs to measure the flexion/extension angles of the hips and knees.

The control system is based on the board LaunchXL-F38377S (Texas Instrument, USA) that runs the control algorithm and acquires sensor data at $1 \mathrm{kHz}$ (excepting IMUs whose sample frequency is $50 \mathrm{~Hz}$ ). The system can be used in a tethered version or a portable set-up where the exoskeleton's control electronics and a Li-Po battery are embedded in a backpack that the user carries. The total weight for the power and electronic system is about $3 \mathrm{~kg}$.

\section{Gait assistive control strategies for the REFLEX prototype}

The main aim of the REFLEX prototype is to deliver assistance to the paretic leg of hemiparetic subjects according to the movement of the non-paretic one. As an example, Panel A of Figure 2 shows the gait pattern of both lower limbs for a healthy subject and a hemiparetic patient. Although there are little differences in the functional gait pattern of both limbs in the healthy subject (as reported by Sadeghi et al. [31]), these are more significant in the hemiparetic patient. In panel A of Figure 2, we also represent the continuous gait phase, which is a function 
142 previous works, the phase of the movement of both limbs is approximately delayed $180^{\circ}$ in 143 healthy subjects $[32,33]$.

\section{A. Examples of healthy and hemiparetic gait patterns}
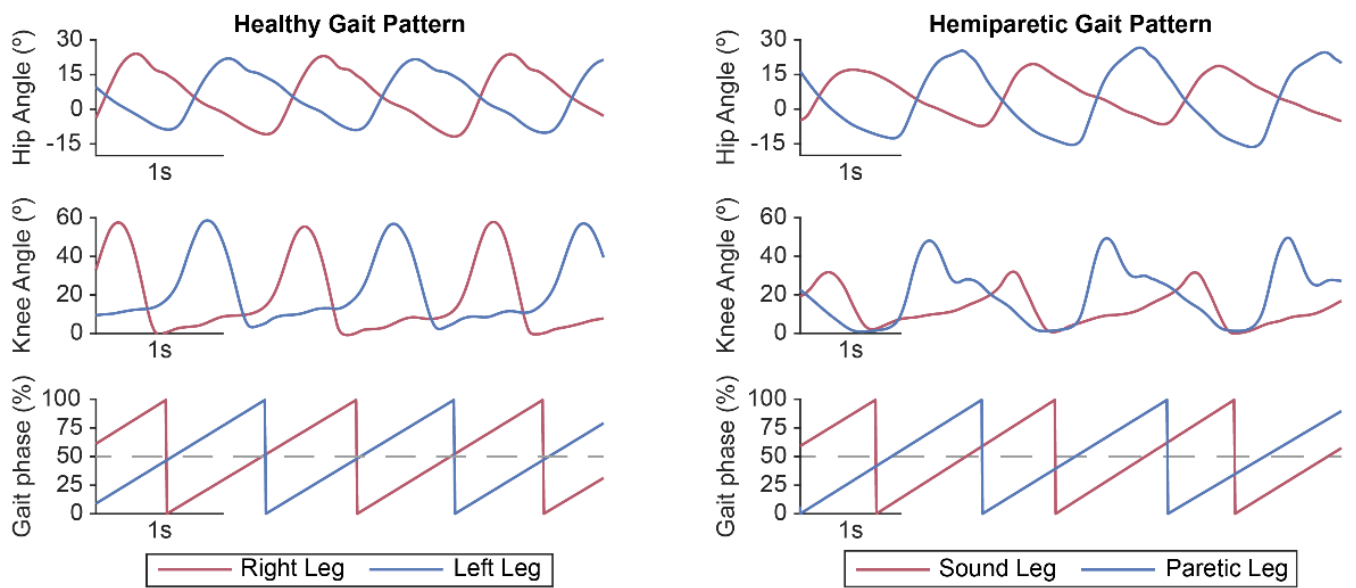

B. Control paradigm overview

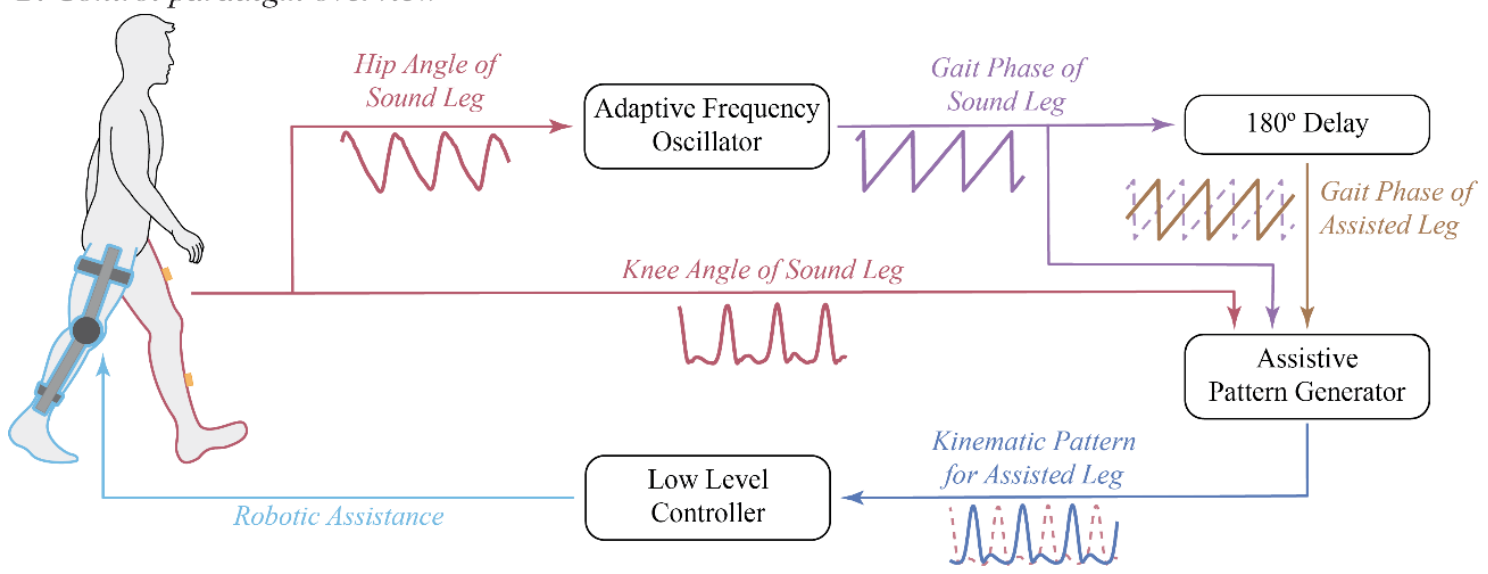

Figure 2: Control paradigm. Panel A. shows examples of healthy and hemiparetic gait patterns. Panel B shows an overview of the control algorithm. The assistance provided by the robotic exoskeleton is synchronized with the movement of the sound leg. The sound hip angle feeds an Adaptive Frequency Oscillator (AO) to estimate the sound leg's gait phase in real-time. This phase is delayed $180^{\circ}$ to obtain the gait phase for the assisted leg. The gait phase of both legs and the sound knee movement is used to generate the pattern to be followed by the exoskeleton through the low-level controller to assist the movement of the assisted limb. 
on the kinematics of the sound leg. Thirdly, the low-level controller generates a force-tunnel around this desired kinematics pattern to assist the limb.

151

152

153

\section{Real-time gait phase estimation}

We use an AO to estimate the real-time gait phase and thus synchronize the REFLEX prototype with the user's healthy side movement [34,35]. Compared with other published control paradigms that synchronize the robot's action, like Finite-State-Machines (FSM) [3639] or EMG-based controllers [40-43], AOs present several advantages. Compared with FSM controllers, AOs generate continuous signals instead of discrete trigger events, enabling more versatile strategies and the use of these signals in the low-level controllers. Besides, compared with EMG controllers, the required sensory system is simpler and more robust.

AOs are dynamic systems that can be synchronized to periodic signals by learning their features as state variables [44]. Considering the hip flexion angle of the sound leg, $\theta_{s}$, as input, an AO can estimate the phase of the sound leg $\varphi_{s}(t)$ according to the next dynamic system [34]:

$$
\varepsilon(t)=\theta_{s}(t)-\hat{\theta}_{s}(t)
$$

$$
\dot{\omega}=-v_{\omega} \varepsilon(t) \sin \varphi_{s}
$$

$$
\begin{gathered}
\dot{\varphi}_{s}=\omega-v_{\varphi} \varepsilon(t) \sin \varphi_{s} \\
\dot{\alpha}_{k}=\eta \cos \left(k \varphi_{s}\right) \varepsilon(t) \quad\left(k=0, \ldots, N_{f}\right) \\
\dot{\beta}_{k}=\eta \sin \left(k \varphi_{s}\right) \varepsilon(t) \quad\left(k=0, \ldots, N_{f}\right) \\
\hat{\theta}_{s}=\sum_{k=0}^{N_{f}} \alpha_{k} \cos \left(k \varphi_{s}\right)+\beta_{k} \sin \left(k \varphi_{s}\right)
\end{gathered}
$$

Where $\varphi_{s}$ and $\omega$ are the phase and frequency of the oscillator synchronized with the sound leg; $\alpha_{k}$ and $\beta_{k}$ are the Fourier coefficients used for estimating $\hat{\theta}_{s}$; and $\varepsilon(t)$ is the 
error in this estimation. $v_{\omega}$ and $v_{\varphi}$ are learning constants and $\eta$ is a coupling factor that determine the dynamic response of the error $\varepsilon(t)$. At every time step, a new input $\theta_{s}$ is considered, and it is used to update all the variables involved in the $\mathrm{AO}$.

The gait phase $\varphi_{s}$ should be a variable that increases monotonically and is reset when the gait cycle is completed. However, the convergence of the AO may not fulfill the criteria of $\varphi_{s}=0$ at heel strike. A phase offset correction is introduced to ensure that $\hat{\varphi}_{s}=0$ at heel strike and give kinematic meaning to the gait phase estimation. According to [45], a phase correction was implemented, so an offset in the phase estimation $\rho$ is updated every time the insole pressure sensors detect the heel-strike event. This offset is low-pass filtered with a first-order Butterworth filter with a cut-off frequency of $0.5 \mathrm{~Hz}$ to avoid abrupt changes in the phase estimation due to phase correction.

$$
\rho=\varphi_{s_{\text {HeelStrike }}}
$$

$$
\hat{\varphi}_{s}=\varphi_{s}-\rho
$$

Once the AO estimates the corrected phase of the sound leg, we calculate the phase of the assisted leg $\varphi_{a}$ by delaying it $\pi \mathrm{rad}$ according to the following equation.

$$
\varphi_{a}=\hat{\varphi}_{s}+\pi
$$

For convenience to gait analysis, the following changes of variables are performed:

$$
\phi_{S}(t)=\frac{\hat{\varphi}_{s}(t)}{2 \pi} \cdot 100
$$

$$
\phi_{a}(t)=\frac{\varphi_{a}(t)}{2 \pi} \cdot 100
$$

$$
f(t)=\frac{\omega(t)}{2 \pi}
$$



frequency in steps per second.

\section{Assistive Pattern Generators.}

Based on the estimation of the gait phase of both legs, the prototype generates an assistive pattern to render a given trajectory to the paretic leg that should contribute to improving gait symmetry. To do so, we developed two different assistive strategies, namely, the Echo-control strategy, which aims to replicate the average movement of the sound leg, and the Adaptive healthy pattern strategy, which aims to synchronize the application of a healthy reference from the literature.

\section{A. Echo-control assistive strategy.}

Figure 3 illustrates the concept of the Echo strategy, which aims at replicating the movement of the sound leg of the user [46]. In the first stage, we consider the movement of the sound leg, so the knee flexion/extension angle is mapped over the gait phase estimated by the AO. By using linear interpolators, the system reconstructs the step kinematics regularly separated by $2 \%$ of the step cycle. This information is stored in a buffer that keeps the kinematics of the last five steps; thus, the average sound pattern is calculated as the mean of this buffer's content.

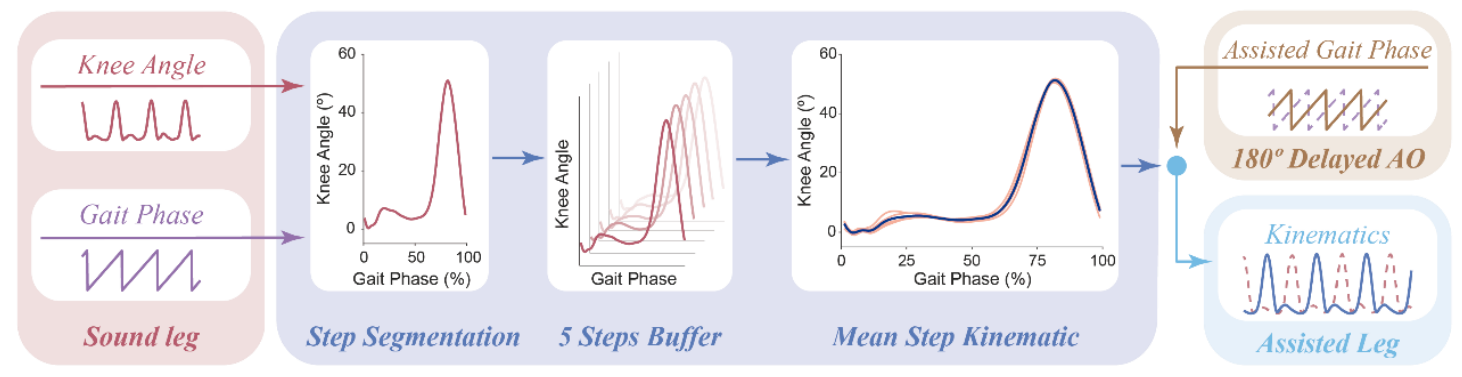

Figure 3: Pattern generation according to the Echo-control assistive strategy based on replicating the kinematics of the sound leg. The knee movement during a step is stored in a five-steps buffer and used to calculate the mean pattern of the sound knee; afterward, this averaged movement is provided as the targeted reference for the robotic exoskeleton according to the gait phase estimated for the assisted limb. 
209 filter designed for a sampling frequency of $50 \mathrm{~Hz}$ (the sampling frequency of the signal if the 210 step has a duration of one second) and a cut-off frequency of $10 \mathrm{~Hz}$ (the fifth part of the 211 sampling frequency). Once the signal is smoothed, it is derived to yield the velocity and acceleration patterns for the flexion movement, according to the following equations:

$$
\dot{\theta}_{r e f}=\frac{d \theta_{r e f}}{d t}=\frac{d \theta_{r e f}}{d \phi_{a}} \cdot \frac{d \phi_{a}}{d t}
$$

$$
\ddot{\theta}_{r e f}=\frac{d^{2} \theta_{r e f}}{d t^{2}}=\frac{d^{2} \theta_{r e f}}{d \phi_{a}^{2}} \cdot\left(\frac{d \phi_{a}}{d t}\right)^{2}
$$

217 kinematic patterns (angle, velocity, and acceleration).

\section{B. Adaptive healthy pattern assistive strategy.}

The healthy pattern strategy uses the information of the sound leg to adapt a reference reference trajectory for robotic gait support published by Koopman et al. [47].

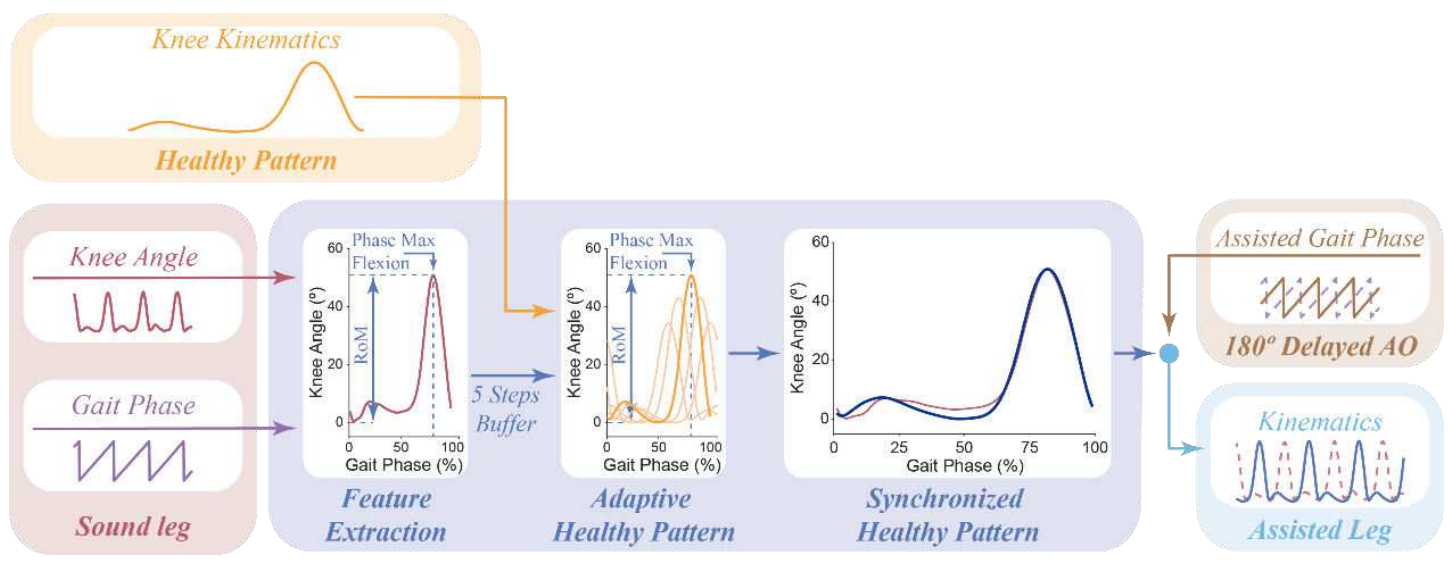

Figure 4: Assistive pattern generation based on synchronizing a healthy gait pattern. The knee pattern is scaled and shifted according to the features extracted from the movement of the sound leg; afterward, it is provided as the set point for the robotic exoskeleton according to the gait phase estimated for the assisted limb. 
This strategy analyzes the kinematic pattern of the sound leg to extract the range of motion and the phase of maximum knee flexion during each step. These features are stored in a five-step buffer, so the average features of the last five steps are used to scale and shift the reference pattern. First-order low-pass Butterworth filters with a cut-off frequency of $0.5 \mathrm{~Hz}$ smooth these factors previously to their application to avoid abrupt changes.

Like the previous strategy, velocity and acceleration references are also generated. In this case, the first and second derivatives of the angular healthy pattern with respect to the gait phase are computed offline, and, afterward, they are scaled and shifted by the same factors as the angle reference. Applying equations (13) and (14), these derivatives with respect to the gait phase are changed to the time domain to be used as velocity and acceleration references for the assisted leg. Once these reference patterns are fully defined, cubic splines are used to interpolate the values for $\theta_{r e f}, \dot{\theta}_{r e f}$ and $\ddot{\theta}_{r e f}$ according to the desired gait phase for the assisted movement.

\section{Variable impedance low-level controller}

The assistance provided by the exoskeleton is based on a variable impedance model that aims to control the interaction between robot and wearer [48]. As in previous related works $[29,49,50]$, the controller used in the prototype has a twofold objective depending on the current gait phase:

1. During the stance phase, the robot aims to reinforce the limb so the system composed of the leg and the exoskeleton can load the user's weight and not collapse. A high impedance model is responsible for this reinforcement since it avoids high deviations from the assistive kinematic pattern.

2. During the swing phase, the robot guides the limb's movement according to the Assisted-As-Needed (AAN) paradigm. The computed error between the reference kinematics and the actual movement is the input for the impedance model that defines the torque applied by the device to assist the user gait. No torque is applied if the 
A. Variable impedance low-level controller

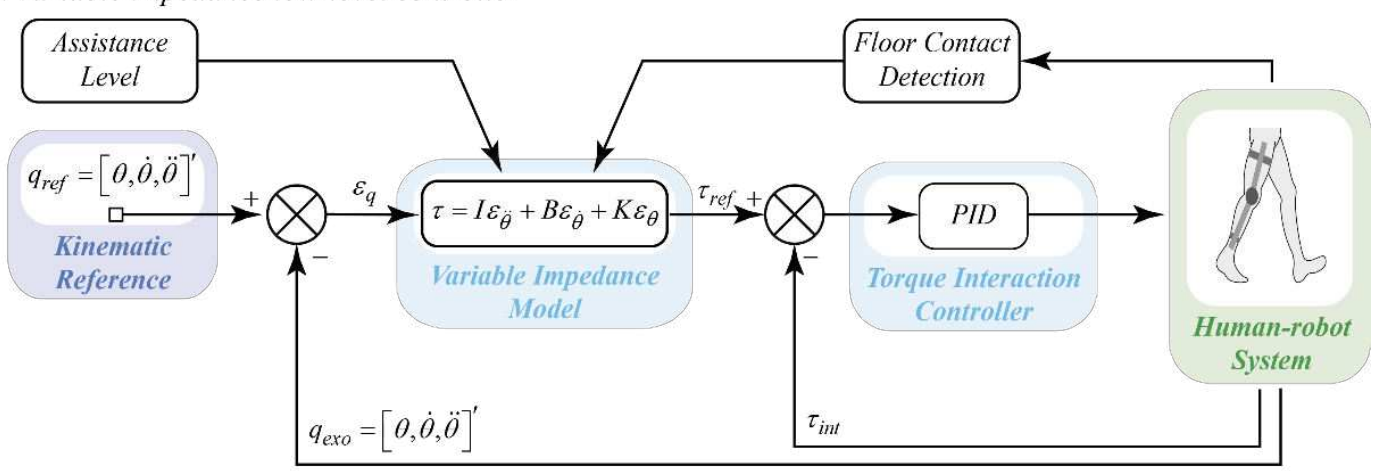

B. Assistance strategy based on force tunnel

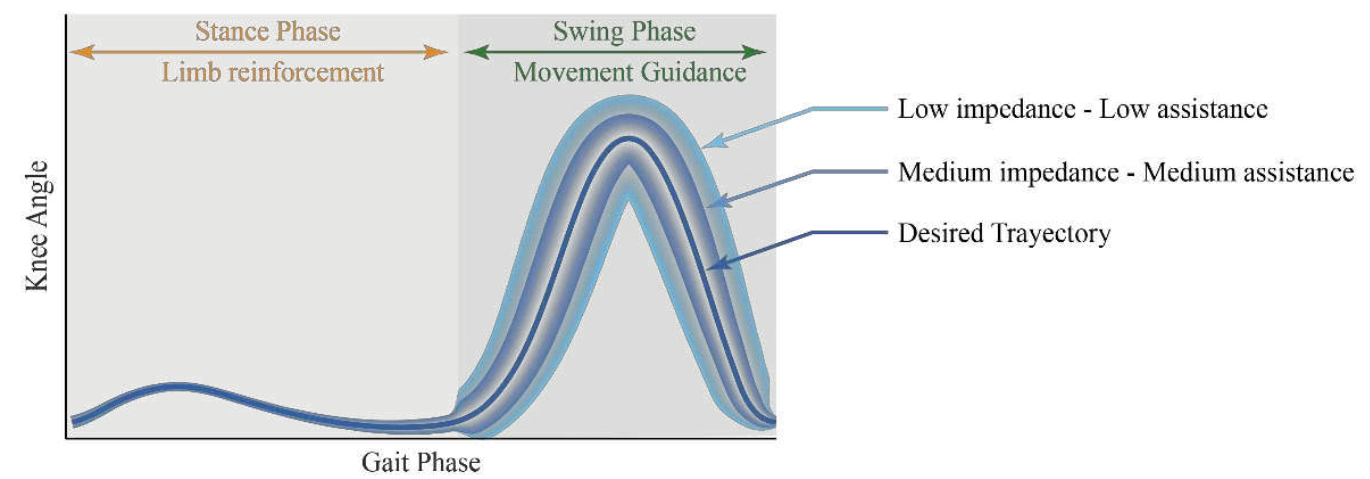

Figure 5: Low-level controller of REFLEX. Panel A shows the block diagram of the variable impedance controller; this controller assists the knee movement following the kinematic reference and according to an Assisted-As-Needed paradigm. Panel B shows the two assistance strategies followed during a single step: the exoskeleton reinforces the joint during the stance phase while it guides the movement during the swing phase; following the Assisted-As-Needed paradigm, the exoskeleton is able to provide different assistance levels by using different force tunnels as depicted in the image.

error is null, while higher errors suppose higher assistive torques. The impedance model depends on the assistance level selected by the therapist or the user, so the force tunnel around the desired trajectory can be changed according to the user's needs.

According to the force-tunnel paradigm, the impedance model calculates the interaction torque that the exoskeleton should provide due to the angular reference tracking error. The system uses a PID controller to follow this torque interaction reference and provide it to the user

(Figure 5).

\section{Experimental validation}

The validation of the REFLEX prototype was implemented in 3 phases. First, we implemented the technical validation of the controller's performance, assessing the phase 
estimation provided by the $\mathrm{AO}$ and the pattern generated by the two assistive strategies. Then,

260 in the second phase, we tested the device with healthy subjects to ensure its proper operation

261 during human interaction. Finally, in the third phase, the prototype was evaluated with stroke 262 patients. subjects ( 3 males, age: $24.7 \pm 3.8$ years, height: $1.78 \pm 0.02 \mathrm{~m}$, weight: $77.7 \pm 2.5 \mathrm{~kg}$; mean \pm standard deviation) and three chronic stroke patients (demographic data are summarized in Table 1). All subjects gave their informed consent for the experiment; the study was conducted in accordance with the Declaration of Helsinki, and it was approved by the local Ethics Committee. Subjects were instructed to walk on a treadmill at a constant speed during trials of 5 minutes. All subjects carried out four different kinds of trials: (1) NoExo: subjects only wore the inertial sensors and the insole pressure sensors to acquire their basal motion; (2) Free: subjects wore the exoskeleton although the actuator was mechanically decoupled, so it enabled the free movement of the knee; (3) Echo: the device provided gait assistance following the Echo-control strategy; and (4) Pattern: the device provided gait assistance following the Pattern strategy. During trials, subjects used the tethered version of REFLEX, and the stroke patients wore a safety harness that did not support any weight. Previously to the execution of the trials, the gait velocity was self-selected by the subjects to a comfortable level.

Table 1: Stroke subjects' demographic data

\begin{tabular}{l|lllllll} 
Ident & $\begin{array}{l}\text { Age } \\
(\text { years })\end{array}$ & $\begin{array}{l}\text { Heigth } \\
(\mathrm{m})\end{array}$ & $\begin{array}{l}\text { Weigth } \\
(\mathrm{kg})\end{array}$ & $\begin{array}{l}\text { Time after } \\
\text { stroke } \\
\text { (months })\end{array}$ & Sex & $\begin{array}{l}\text { Hemiparetic } \\
\text { side }\end{array}$ & Stroke \\
\hline$P 1$ & 63 & 1.8 & 83 & 11 & Male & Left & $\begin{array}{l}\text { Cortical } \\
\text { ischemic }\end{array}$ \\
$P 2$ & 57 & 1.72 & 84 & 7 & Male & Right & $\begin{array}{l}\text { Subcortical } \\
\text { hemorrhagic } \\
\text { Cortical }\end{array}$ \\
$P 3$ & 54 & 1.72 & 60 & 9 & Male & Left & ischemic \\
\hline $\begin{array}{l}\text { Average } \\
{ }^{1} \text { Mean } \pm \text { standard deviation }\end{array}$ & $\begin{array}{l}58 \pm 4.6 \\
1.74 \pm 0.05\end{array}$ & $75.6 \pm 13.6$ & $9 \pm 2$ & & &
\end{tabular}



seconds at least.

Subjects rested between trials for five minutes at least to avoid adaptation and learning effects from trial to trial. In addition, in NoExo, Free, Echo, and Pattern trials, only the two last minutes of the trials were processed to evaluate the gait once the steady-state was reached. For the process of the data, we considered the beginning of each step when the insole pressure sensors detected the heel strikes. All experimental data were recorded at $50 \mathrm{~Hz}$.

When data distributions were compared, they were composed of the metrics calculated in each step during an experimental condition. After checking the non-normality of the data (Kolmogorov-Smirnov test; $\mathrm{P}<0.005$ ) and the heteroscedasticity (Levene test; $\mathrm{P}<0.005$ ), we looked for significant differences between experimental conditions (Kruskall-Wallis test; $\mathrm{P}<0.005)$.

\section{Results}

\section{Technical Validation}

For the technical validation of REFLEX, we evaluated its performance on healthy subjects. First, we assessed the estimation of the continuous gait phase and frequency performed by the AO; afterward, we generated offline the assistive pattern based on VariableSpeed and NoExo data and compared them with the actual movement of the subjects.

\section{AO validation} time phase and frequency of the gait. We compared these results with the real gait phase and frequency computed offline according to the heel strike events.

Figure 6 illustrates the $\mathrm{AO}$ estimations for one trial; panels $\mathrm{A}$ and $\mathrm{C}$ show how the $\mathrm{AO}$ adapted its estimations to velocity changes. Table 2 summarizes the error for the phase and 
A. Gait phase estimation

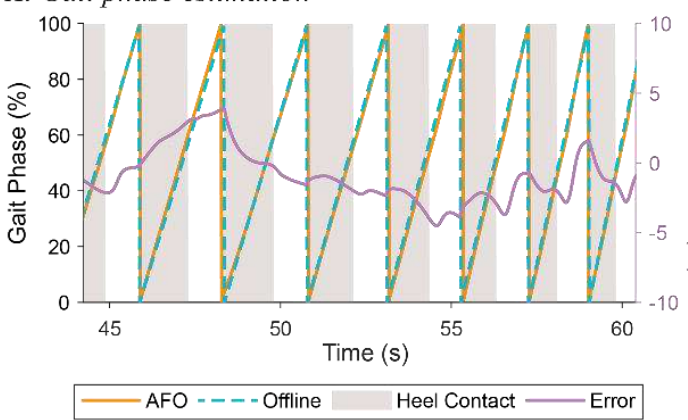

B. Average gait phase estimation

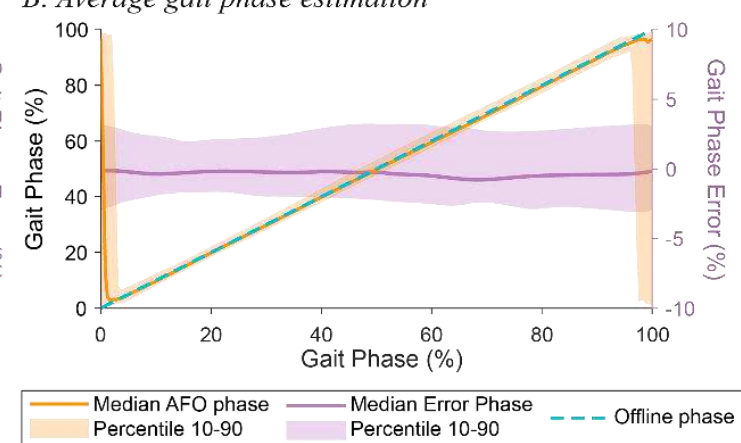

C. Gait frequency estimation

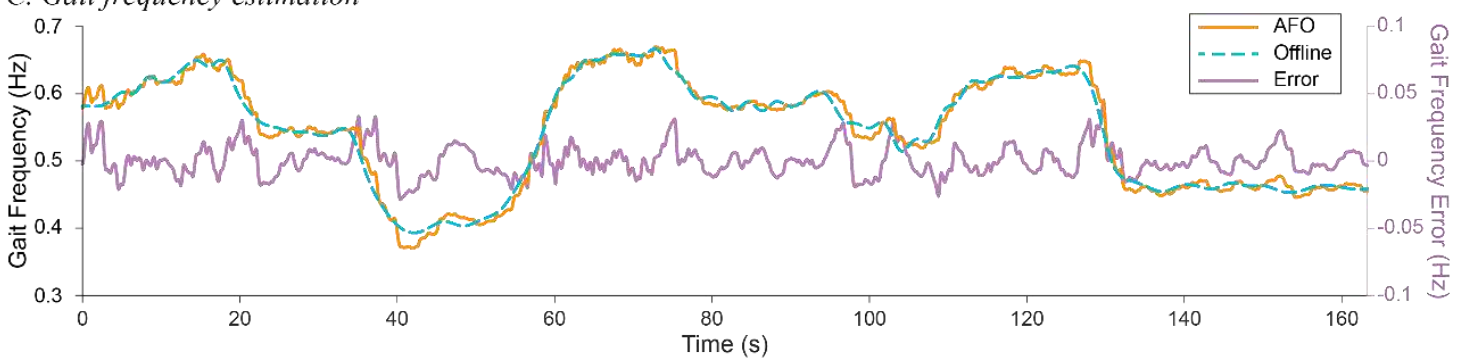

Figure 6: Example of AO results during one healthy subject trial walking at variable gait velocity. Panel A compares the AO phase estimation (solid orange line) with the offline phase calculated based on the heel strike detected by the insole pressure sensors (dashed cyan line) during a trial segment. Panel B shows the median phase estimation during a step in the trial and median error during the step; areas represent the 10-90 percentiles. Panel

$C$ compares the gait frequency estimated by the AO in real-time (solid orange line) with the gait frequency calculated offline based on heel strike (dashed cyan line) during the trial. For all the panels, purple lines show the error between the AO estimation and the offline result and are represented with respect to the right axis. RMS error (RMSE) is lower than $2.4 \%$ and frequency RMSE is lower than $0.015 \mathrm{~Hz}$.

\section{Assistive pattern generation}

For healthy subjects, we considered the left leg as the master leg (correspondent to the sound leg in hemiparetic subjects, i.e., the leg that is the reference for the pattern generation), so the generated reference should be synchronized with the movement of the right leg (also named equivalent leg). Panel A of Figure 7 shows the kinematic pattern generated by both assistive strategies during a portion of one VariableSpeed trial; it is remarkable how the controller

Table 2: Errors in gait phase and frequency estimation

\begin{tabular}{l|c|c} 
& Average Error & RMSE \\
\hline Gait phase $(\%)$ & $-0.28 \pm 2.36$ & 2.37 \\
Frequency $(\mathrm{Hz})$ & $-2.16 \mathrm{e}-04 \pm 0.014$ & 0.014 \\
${ }^{I}$ Mean \pm standard deviation
\end{tabular}


A. Example of knee flexion and Set Point generation
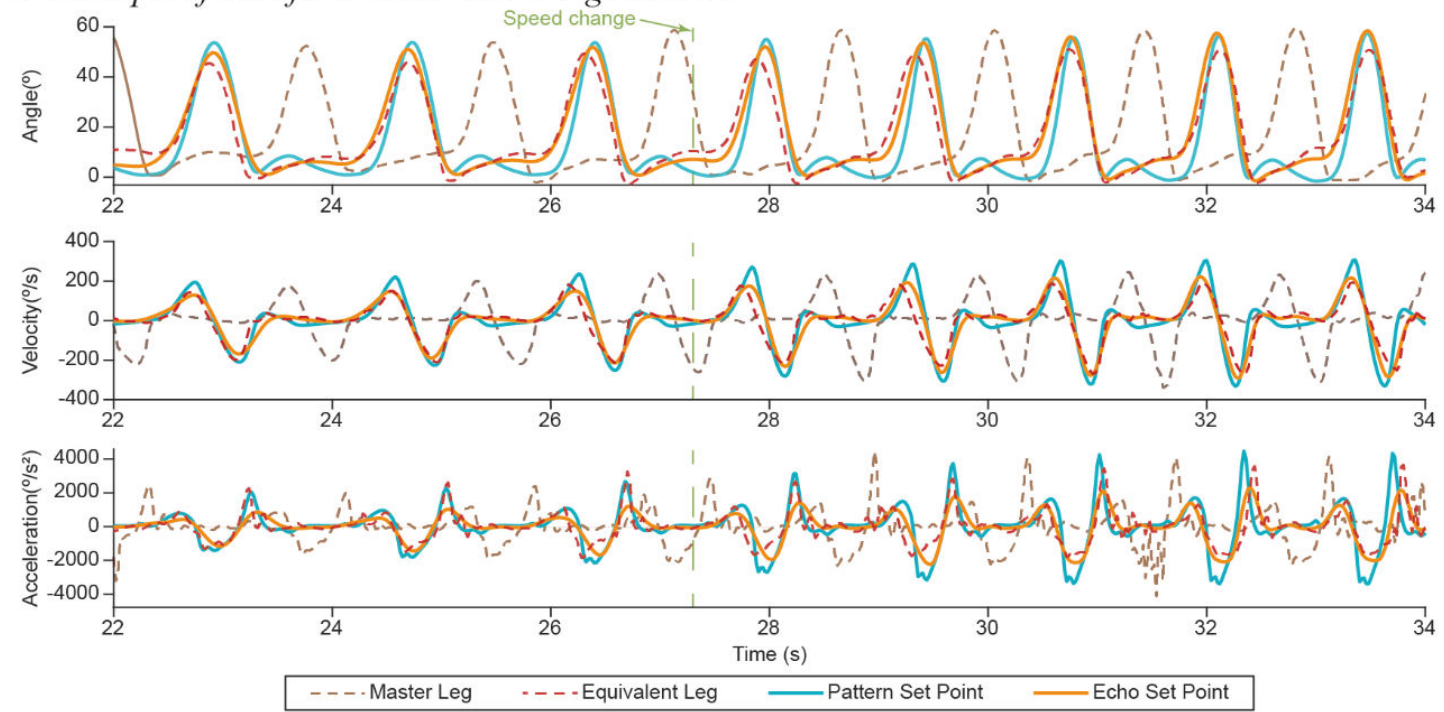

B. Phase portraits of master knee flexion and set point generation
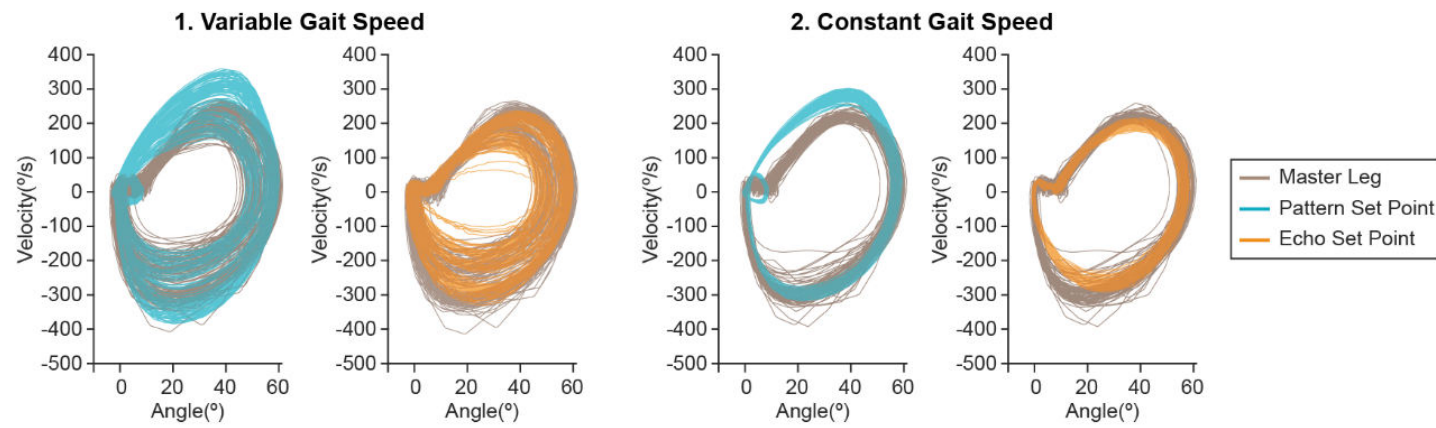

Figure 7: Example of set point generation with the experimental data of one healthy subject. Panel A shows an example of the set-point calculated by the two assistive strategies (Pattern in blue and Echo in orange); they are compared with the movement of the master leg that fed the algorithm (brown dashed line) and with the movement of the equivalent leg whose motion should be synchronized with the generated set point (red dashed line). Panel B compares the phase portrait of the movement of the master leg (in brown) with the set point generated by the Pattern assistive strategy (in blue) or the echo assistive strategy (in orange). Two experimental conditions were evaluated: variable gait speed (two left panels) and constant gait speed (two right panels).

reacted to gait velocity changes with a minimum loss of synchrony.

Panel B of Figure 7 shows the phase portraits of the generated pattern and the master leg's motion used as the basis. This representation shows the angular position in the $\mathrm{X}$-axis and the angular velocity in the Y-axis, so the resulting portrait's shape is representative of the dynamics of the motion [51]. Notice that during the trials at variable gait speed, the dispersion of the phase portraits is higher than for the trials at a constant speed. We adopted three metrics to evaluate the pattern generated with both assistive strategies, aiming to assess its waveform and timing: 
A. Phase portrait simmilarity Set Point vs Master Leg

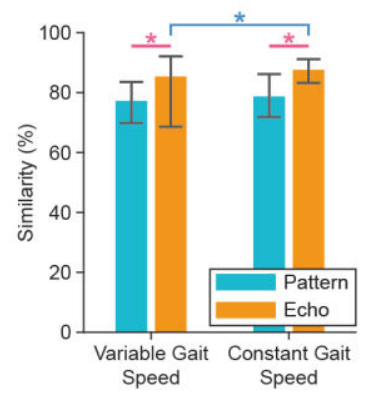

B. Correlation Set Point vs Equivalent Leg

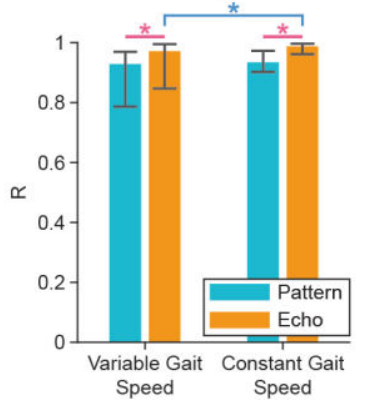

C. Meximum Flexion Delay Set Point vs Equivalent Leg

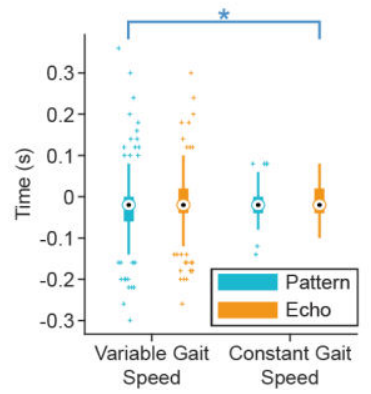

Figure 8: Assessment of the set-point generated by the two assistive strategies. Panel A shows the phase portrait

1) Similarity between the generated pattern and the movement of the master leg. To evaluate it, we assess the similarity between their phase portrait, according to the following metric:

$$
\operatorname{Similarity}(\%)=\frac{A \cap B}{A \cup B} \cdot 100
$$

where $A$ and $B$ are the areas of two phase portraits, so $A \cap B$ is the common area between them and $A \cup B$ is the union of both areas. Panel A in Figure 8 represents the distributions of this metric, and Table 3 summarizes their average value. The average similarity of the assistive pattern with the master leg's motion is $77.5 \pm 5.92 \%$ for Pattern strategy and $84.31 \pm 8.43 \%$ for Echo strategy. 
similarity between the set-point and the movement of the master leg used for generating it. Panel B shows the correlation between the set-point and the movement of the equivalent leg (a correlation of 1 means a perfect synchronization). Length bars indicate the median value and whiskers the 10-90 percentiles. Panel C shows the boxplot of the delays between the maximum knee flexion between the set point and the movement of the equivalent leg. Markers (*) show significant differences between experimental conditions.

Table 3: Phase portrait similarity (\%) between assistive pattern and master leg.

\begin{tabular}{l|c|c} 
& Pattern & Echo \\
\hline Variable Gait Speed & $77.03 \pm 5.98$ & $82.52 \pm 10.03$ \\
Constant Gait Speed & $78.57 \pm 5.63$ & $87.22 \pm 3.40$ \\
\hline Global & $77.63 \pm 5.89$ & $84.35 \pm 8.44$ \\
Mean \pm standard deviation
\end{tabular}

2) Timing of the generated pattern. To this end, we compared this pattern with the movement performed by the equivalent leg. We evaluated this timing twofold: checking the correlation between both movements and assessing the maximum knee flexion delay in both patterns. In Figure 8, panels B and C represent the distribution of these metrics, and Tables 4 and 5 summarize their average values. For the Pattern strategy, the average correlation is $\mathrm{R}=0.9 \pm 0.1$, and the average delay is $-0.021 \pm$ $0.060 \mathrm{~s}$, while the Echo strategy resulted in a correlation of $\mathrm{R}=0.95 \pm 0.08$ and an average delay of $-0.012 \pm 0.053 \mathrm{~s}$. 
Table 4: Correlation $(R)$ between assistive pattern and equivalent leg.

\begin{tabular}{l|c|c} 
& Pattern & Echo \\
\hline Variable Gait Speed & $0.89 \pm 0.12$ & $0.94 \pm 0.10$ \\
Constant Gait Speed & $0.93 \pm 0.03$ & $0.98 \pm 0.02$ \\
\hline Global & $0.90 \pm 0.10$ & $0.95 \pm 0.08$
\end{tabular}

Table 5: Maximum flexion delay (s) between assistive pattern and equivalent leg.

\begin{tabular}{l|c|c} 
& Pattern & Echo \\
\hline Variable Gait Speed & $-0.023 \pm 0.071(0.074)$ & $-0.015 \pm 0.063(0.065)$ \\
Constant Gait Speed & $-0.017 \pm 0.037(0.041)$ & $-0.007 \pm 0.034(0.035)$ \\
\hline Global & $-0.021 \pm 0.060(0.064)$ & $-0.012 \pm 0.053(0.055)$ \\
Mean \pm standard deviation (RMS error between brackets)
\end{tabular}

The following results are from the experiments that we conducted to understand how the assistance provided by the exoskeleton affects the gait of healthy subjects. Figure 9 illustrates, as an example, the knee phase portraits during one experimental trial with one healthy subject.

340 Comparing how the phase portraits evolved across trials allows us to visualize how the 341 movement of both knees (actuated and not) changed according to REFLEX operation mode.

342 Panel A in Figure 10 shows the average phase portraits of both legs during trials and how the 343 similarity measured in the NoExo trial decreased due to wearing the robot in the Free trial.

344 However, the action of the robot actively improved similarity during Echo and Pattern trials.
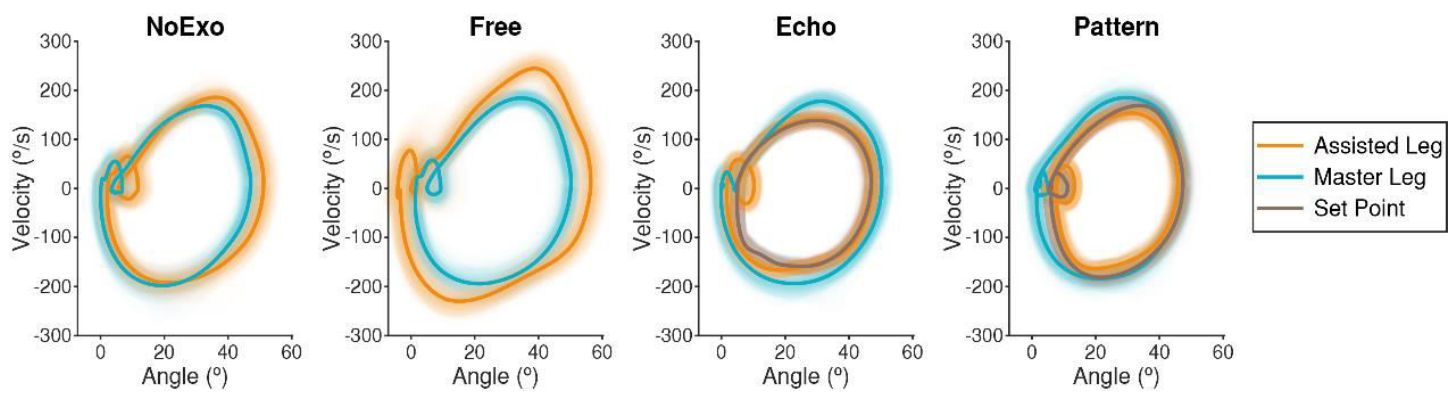

Figure 9: Phase portrait representation of the knee movement from the trials performed by a healthy subject (HS1). Orange lines correspond to the movement of the assisted knee while cyan lines correspond to the movement of the master knee; brown lines correspond to the set point followed by the robot. Solid lines represent the median movement, while semi-transparent lines correspond to the movement of individual steps. When the user wears the robot, the phase portrait of the assisted leg changes in hip and knee joints; however, the action of the robot during

Echo and Pattern conditions compensates the effect of wearing the robot and make the phase portrait of the assisted leg closer to the portrait of the master leg. 
A. Phase portraits of knee flexion for one healthy subject
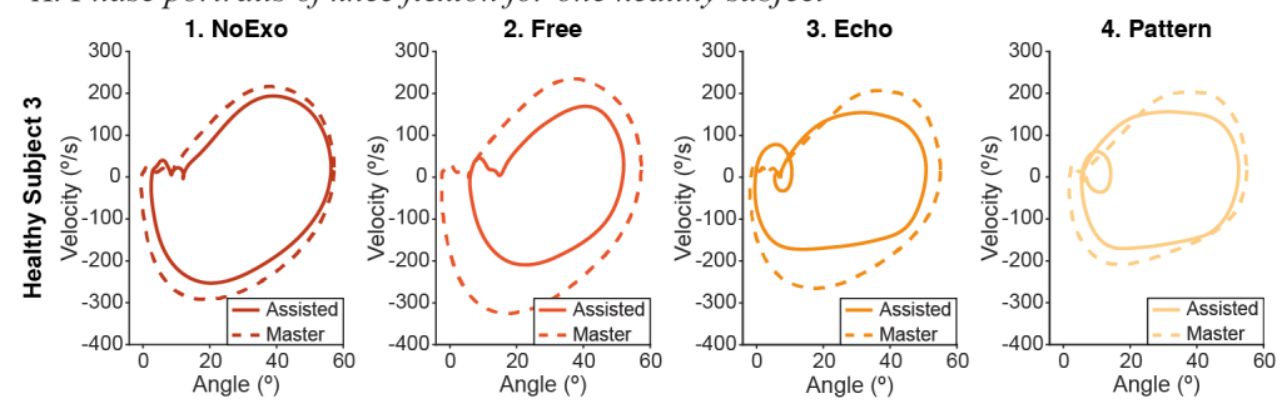

B. Comparison between median knee flexion phase portrait

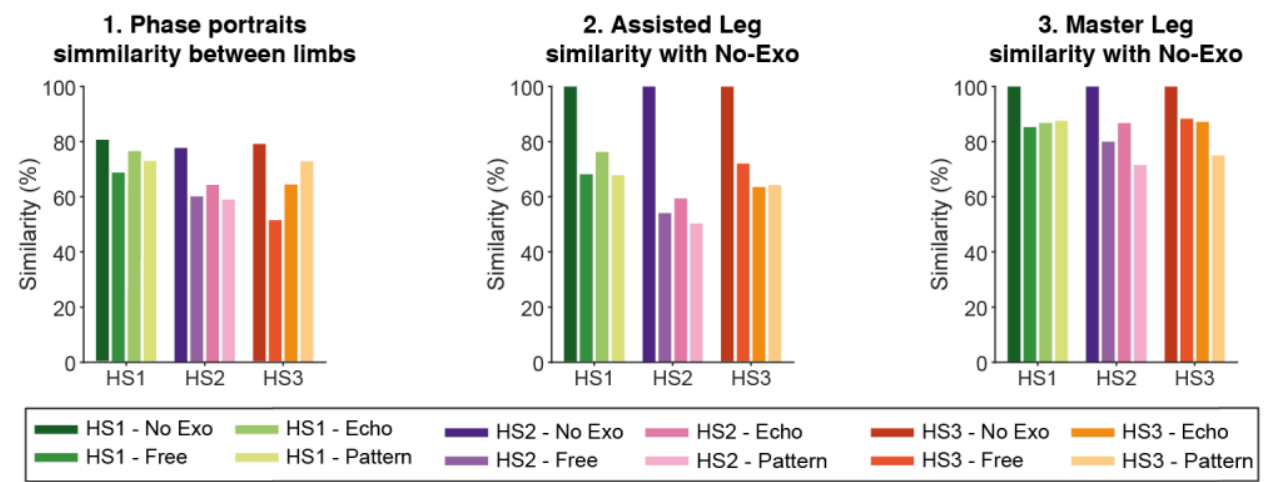

Figure 10: Panel A. shows the median phase portrait for each leg; columns 1-4 include information for each experimental condition. Solid lines represent the assisted leg, and dashed lines represent the master leg. Panels $B$. show the similarity between the areas of the phase portrait: Panel B1 represents the similarity between both limbs under each experimental condition, and panels B2 and B3 represent the similarity between the movement of the current experimental condition and the movement during NoExo for each leg. Across the figure, data for the same patient is represented in the same color (green for HS1, purple for HS2, orange for HS3) while the brightness

indicates the experimental condition (from darkest to lightest: NoExo, Free, Echo, and Pattern). Similarity decreases because of wearing the robot; however, the assistance provided by the exoskeleton actively improves similarity in all patients.

To evaluate the similarity between phase portraits, we used the metric defined in

346 Equation (15). Panel B of Figure 10 shows the results of comparing different median phase

347 portraits according to this metric. Panel B1 represents the comparison between assisted and

348 master motion in each experimental trial. Results pointed out that the same behavior was

349 replicated in all subjects. Wearing the device hampered the similarity between limbs, i.e., the

350 symmetry; however, the provided assistance partially compensated the effect of wearing the

351 device since the similarity increased with respect to the Free condition. Nonetheless, it did not

352 reach the similarity level of the NoExo condition. Only the Pattern strategy in Subject 2 did not

353 improve the similarity with respect to the Free condition. Changes in interlimb similarity were 

panel B3).

In order to evaluate the performance of the assistance, we also assessed the effect of the exoskeleton's action over the range of motion (RoM) of the knee and the gait cycle percent of the maximum knee flexion (Figure 11). We considered the metric value in each step to create a distribution for each subject during each experimental condition.

$$
\operatorname{SI}(\%)=\frac{\bar{X}_{A}-\bar{X}_{S}}{\frac{1}{2}\left(\bar{X}_{A}+\bar{X}_{S}\right)} \times 100
$$

where $\bar{X}_{A}$ and $\bar{X}_{S}$ are the mean value for a metric in the assisted and sound (or master) leg, respectively. A SI of zero value means a complete symmetry, so a higher SI means a higher asymmetry in the metric. The fourth column of Figure 11 shows that the RoM and the phase of maximum knee flexion are more symmetric during assisted trials than during the Free trials. However, although the highest symmetry for the phase of maximum knee flexion was achieved during assisted trials, the highest RoM symmetry is yielded during the NoExo trial.

\section{Validation on hemiparetic patients}

During the third validation phase, we evaluated the performance of the prototype with real hemiparetic patients. To this end, we evaluated how they reacted to the assistance provided by the prototype; an example of the data recorded for a patient is represented in Figure 12. To compare the motion dynamics in both knees, we used the median phase portrait of the flexion/extension movement (Figure 13, panel A). We evaluated their similarity according to Equation (15), showing the results in panel B of Figure 13. 

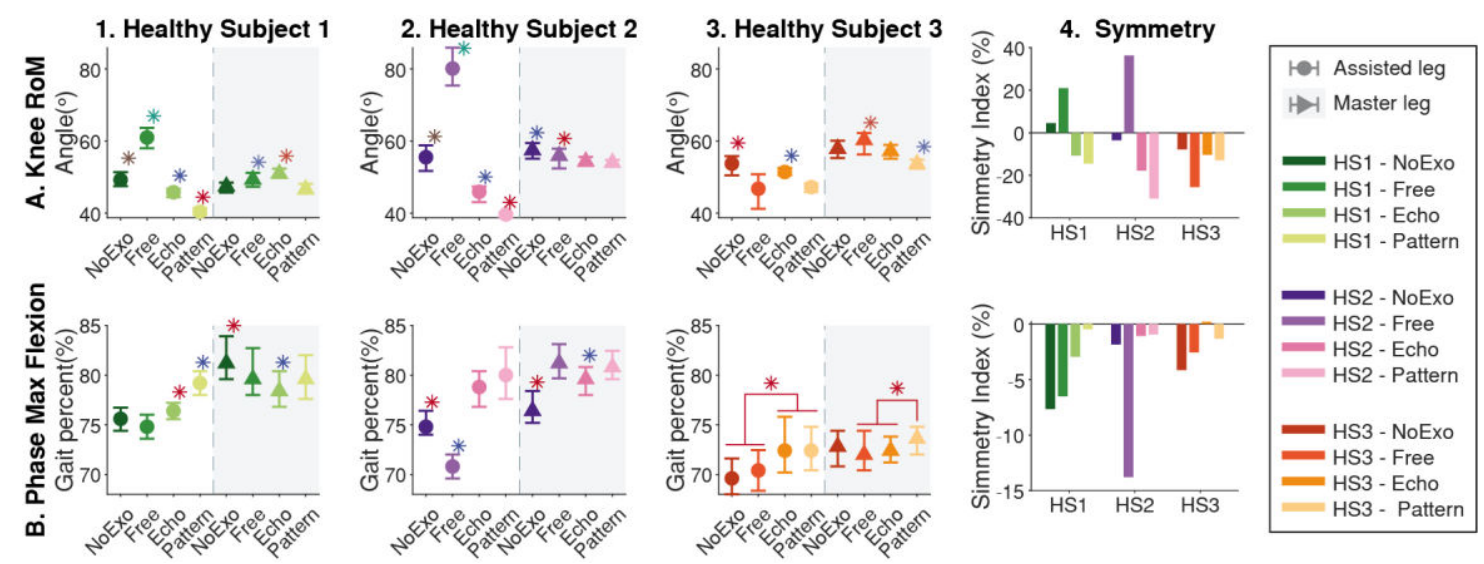

Figure 11: Knee kinematics symmetry for healthy subjects. Rows A. and B. represent the knee Range of Motion and the phase at maximum flexion for the knee. Columns 1-3 represent the result for each subject; markers indicate the median value and whiskers the 10-90 percentiles. Column 4 represents the symmetry index for each subject under each experimental condition; notice that symmetry indexes closer to 0 mean a higher symmetry between limbs. Across the figure, colors represent the same healthy subject (HS1 in green, HS2 in purple and HS3 in orange), the brightness represents the experimental condition (from darkest to lightest: No-Exo, Free, Echo and Pattern correspondingly) and the shape of the marker represents the assessed limb (circle for the assisted limb and triangle for the master leg). Markers (*) show significant differences between experimental conditions within a limb of a subject.

As shown in panel B1 of Figure 13, the robot assistance always improved the similarity between both limbs' motion with respect to the motion in the Free condition. In two out of three patients (P1 and P3), wearing the robot (Free condition) hampered the symmetry in the motion; however, the action of the robot compensated this effect (P1 during Pattern and P3 during Echo) or even increased the symmetry with respect to the NoExo condition (P3 during Pattern). In subject P2, wearing the exoskeleton increased the symmetry, and the exoskeleton's action even increased this improvement. In all subjects, the Pattern strategy led to a more symmetric motion than the Echo strategy. movement during NoExo trials for the assisted and non-assisted legs, respectively. As we can see, both legs' motion changed due to the exoskeleton's action and, therefore, the assistance did not only act over the movement of the assisted leg, but it also affected the movement of the nonassisted leg to attain a more symmetric gait. 

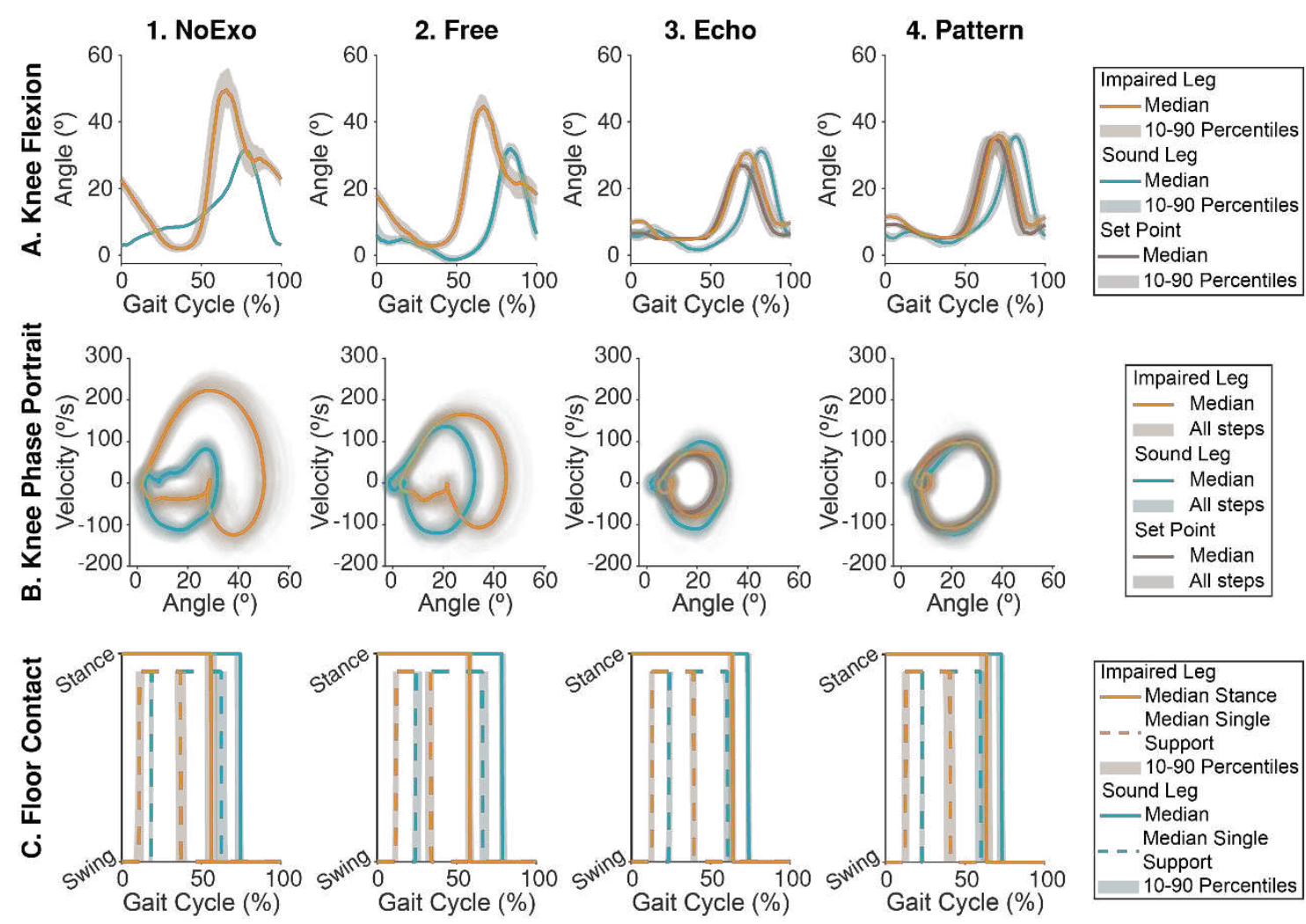

Figure 12: Example of step data from a hemiparetic patient (P2) during the different experimental conditions.

Columns 1-4 include information for each experimental condition: NoExo, Free, Echo, and Pattern correspondingly while rows A-C show the knee kinematics (A), its phase portrait (B), and the foot contact with the floor (C). Information is represented for both legs: the paretic leg is represented in orange while the sound leg is represented in cyan. For the assistive experimental conditions, the exoskeleton set point is also represented in brown.

To evaluate the performance of the controllers, we evaluated the symmetry of the RoM and the phase of maximum knee flexion in both legs (Figure 14) by using the symmetry index defined in the equation (16) (represented in the fourth column of Figure 14). The robot's action yielded more symmetric RoMs in all patients than the Free condition; besides, pattern assistance and echo assistance in P1 and P2 attained a higher RoM symmetry even compared with the NoExo condition. The effect of the mass of the robot was heterogeneous: patients P1 and P3 worsened the RoM symmetry while P2 improved it. The symmetry of the maximum flexion phase decreased because of wearing the exoskeleton in all patients. However, the robot assistance compensated for this effect and improved the symmetry with respect to NoExo in all patients and under all control strategies, except P3 during Echo that seemed not to be affected. 
A. Phase portraits of knee flexion for stroke patients

1. NoExo
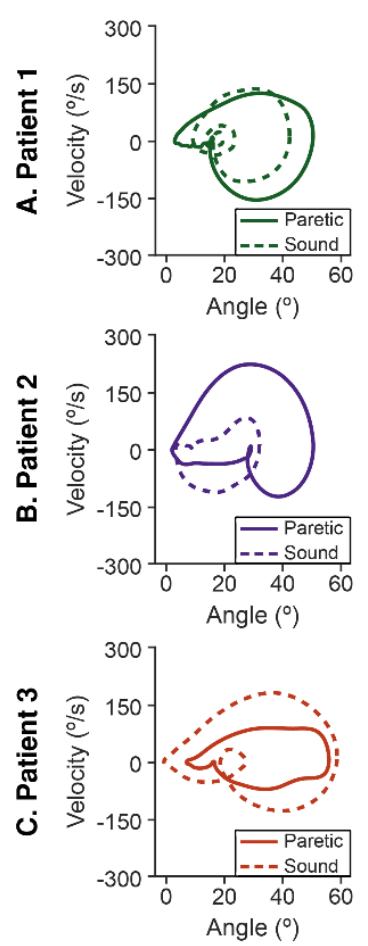

2. Free
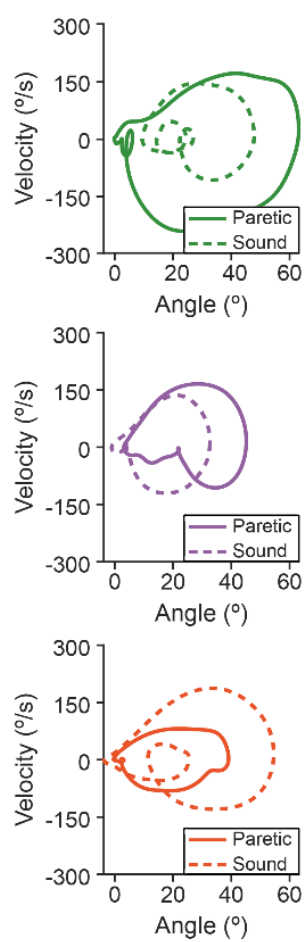

3. Echo
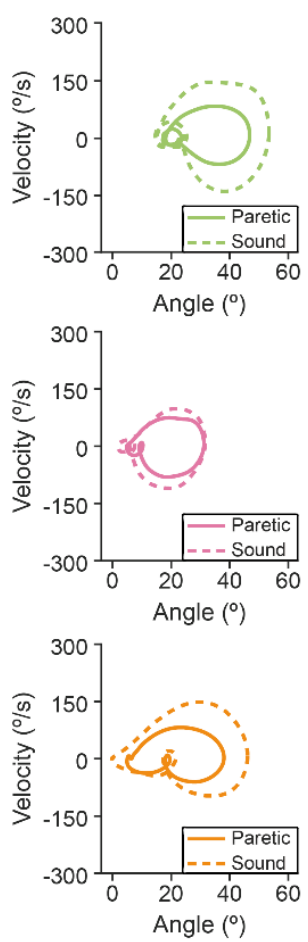

4. Pattern
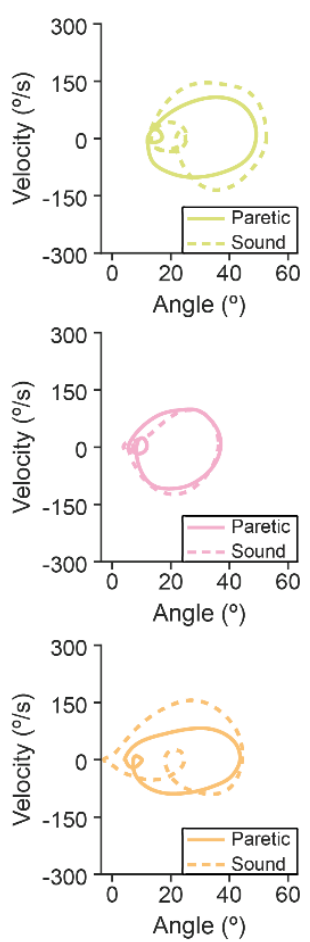

B. Comparison between knee flexion phase portrait

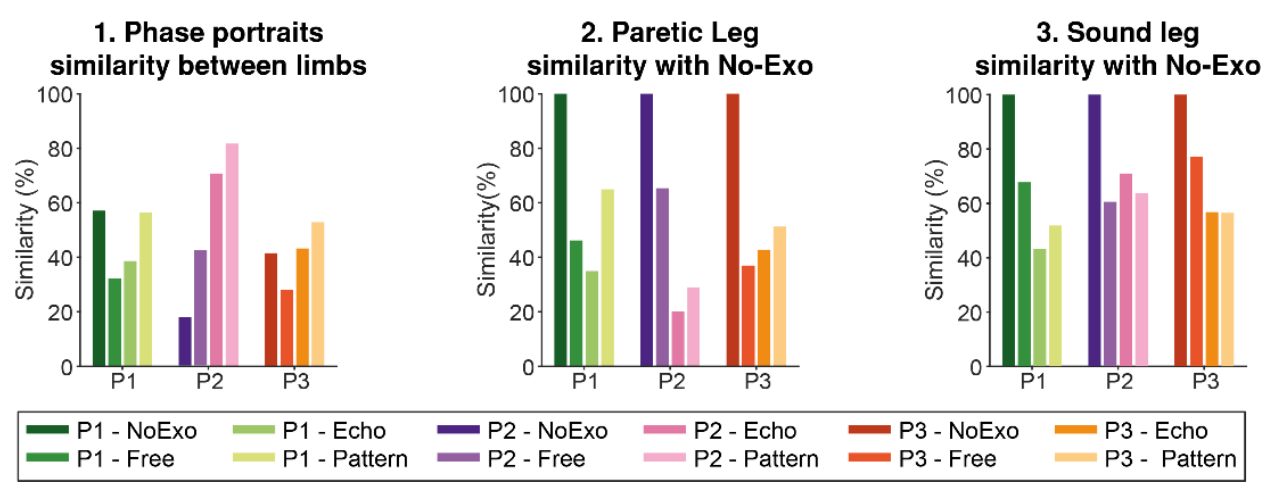

Figure 13: Knee flexion phase portraits for three stroke patients. Panel A. shows the median phase portrait for each limb, columns 1-4 include information for each experimental condition, while rows A-C show the data for the three patients. Solid lines are used for the impaired leg, while dashed lines are used for the sound leg. Panels $B$ show the similarity between phase portraits; Panel B1 represents the similarity between both limbs under each experimental condition, and Panels B2-B3 represent the similarity between the movement of the current experimental condition and the movement during NoExo. Across the figure, data for the same patient is represented in the same color (green for $P 1$, purple for $P 2$, and orange for $P 3$ ) while the brightness indicates the experimental condition (from darkest to lightest: NoExo, Free, Echo, and Pattern).

We also assessed the effect of the assistance on the compensation mechanisms developed by the patients. By using the floor contact information and the IMUs data, we analyzed the influence of the exoskeleton over some gait parameters related to compensatory strategies in 

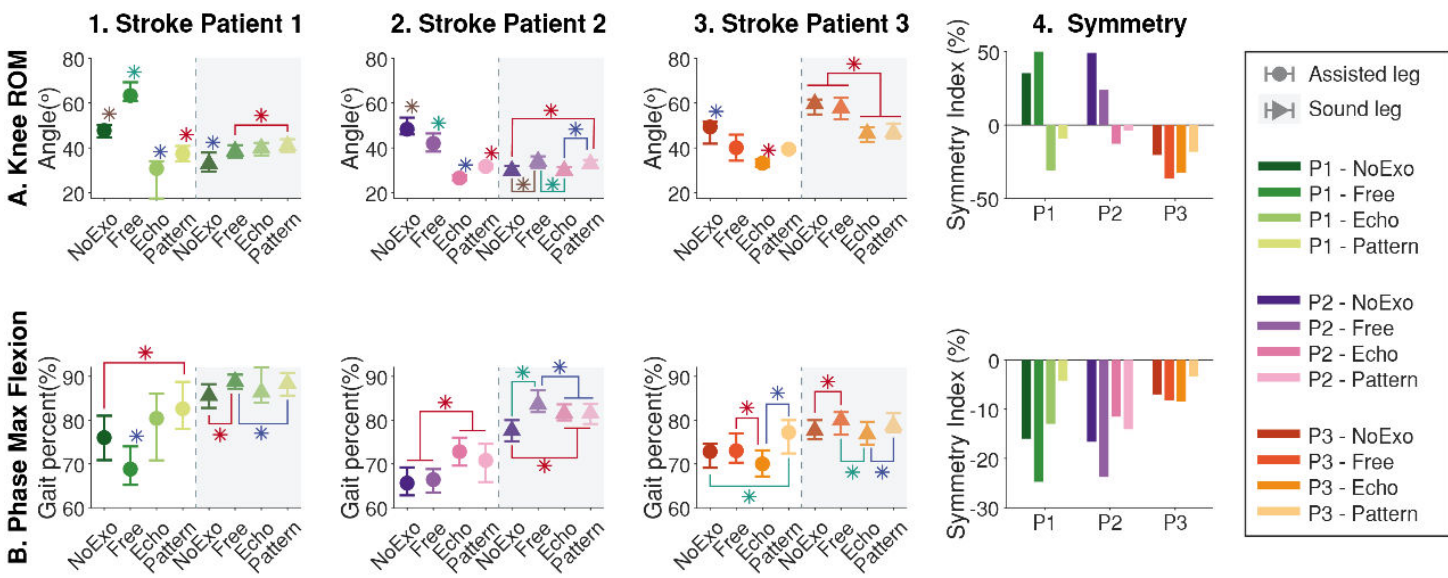

Figure 14: Knee kinematics symmetry for stroke patients. Rows A. and B. represent the knee Range of Motion and the phase at maximum flexion for the knee. Columns 1-3 represent the result for each subject; the markers indicate the median value, and the whiskers indicate the 10-90 percentiles. Column 4 represents the symmetry index for each subject under each experimental condition; notice that symmetry indexes closer to 0 means a greater symmetry between limbs. Across the figure, colors represent the same patient (P1 in green, P2 in purple and P3 in orange), the brightness represents the experimental condition (from lightest to darkest: No-Exo, Free, Echo and Pattern correspondingly) and the shape of the marker represents the assessed limb (circle for the assisted limb and triangle for the sound leg). Markers (*) show significant differences between experimental conditions within a limb

402 hemiparetic gait such as (a) step length, (b) step time, (c) step velocity, (d) single-support 403 duration, (e) stance phase duration, and (f) swing time (rows A-F of Figure 15). 
404 We used the symmetry index to evaluate the action of the robot over these metrics (fourth 405 column of Figure 15). Overall, the assistance provided by the robot led to more symmetric 406 features compared with NoExo trials, or, at least, it compensated for the detrimental effect of 407 wearing the extra mass of the device. However, for some features, patients presented a 408 heterogeneous response.

409 Step length symmetry was improved during all assistive trials compared with the basal 410 condition (excepting Echo in P3). However, each patient responded differently: improvements 411 in P1 were because of wearing the robot (assistive strategies and Free condition yielded the 412 same results), P3 improvements were also because of wearing the device, although the 413 assistance decreased this symmetry (nonetheless, Pattern condition supposed higher symmetry 414 than NoExo condition), and P2 reported symmetry improvements even when wearing the device 415 resulted in its decrease (panel 4A of Figure 15). 

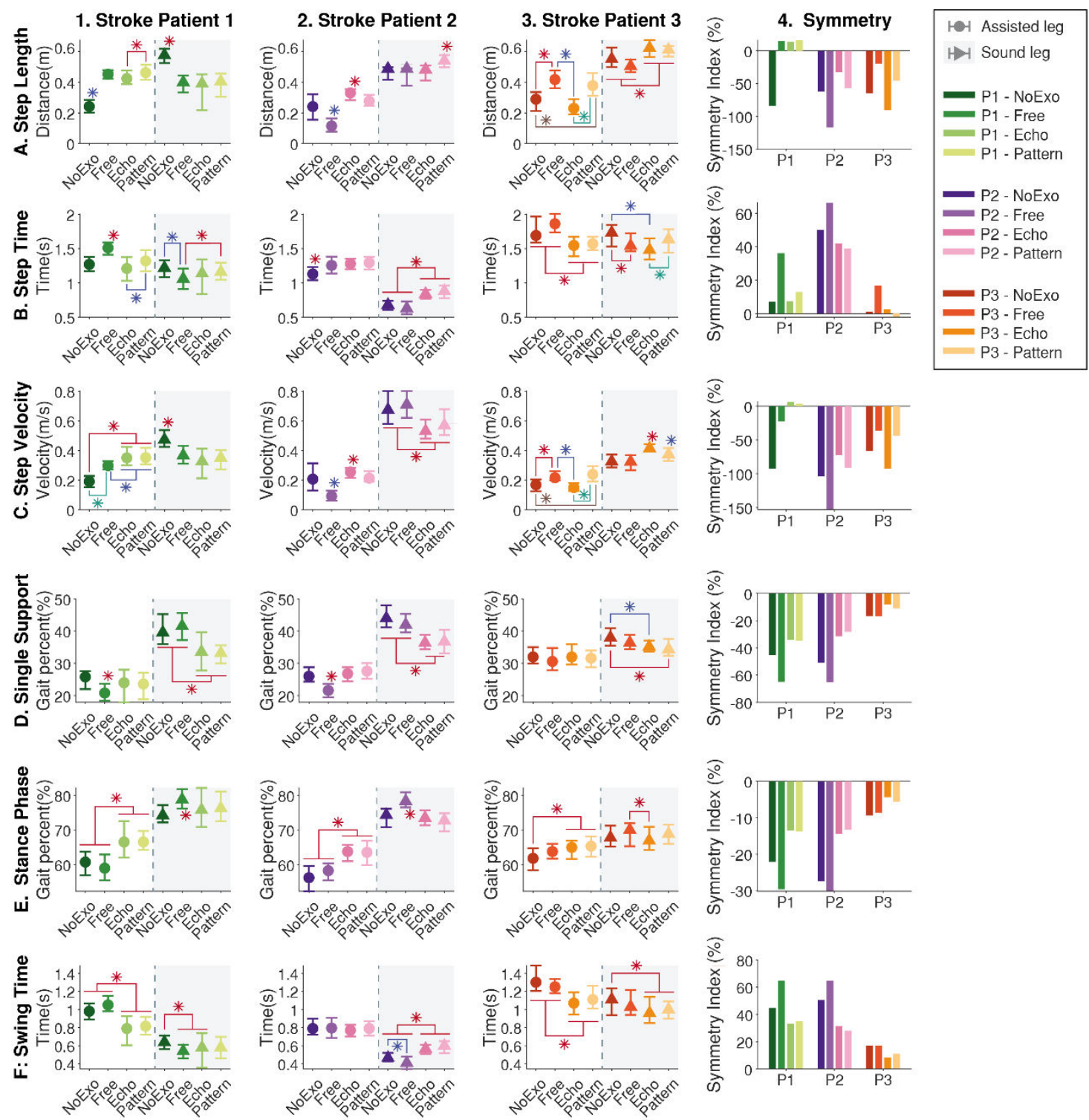

Figure 15: Gait features symmetry for stroke patients. Rows A. to F. include different gait metrics: step length (A),

step time $(B)$, step velocity $(C)$, single-support duration $(D)$, stance phase duration $(E)$, and swing time $(F)$.

Columns 1-3 represent the result for each subject; markers indicate the median value and whiskers the 10-90 percentiles. Column 4 represents the symmetry index for each subject under each experimental condition; notice that that symmetry indexes closer to 0 means a higher symmetry between limbs. Across the figure, colors represent the same patient (P1 in green, $P 2$ in purple and P3 in orange), the brightness represents the experimental condition (from lightest to darkest: No-Exo, Free, Echo and Pattern correspondingly) and the shape of the marker represents the assessed limb (circle for the assisted limb and triangle for the sound leg). Markers (*) show significant differences between experimental conditions within a limb of a subject.

For all patients, wearing the robot supposed a decrease in the step time symmetry (panel 4B of Figure 15). However, this effect was compensated by the action of the assistive strategies, and P2 even increased step time symmetry with respect to his basal condition. Regarding the step velocity (panel 4C of Figure 15), both assistive strategies led to an increase of symmetry in 
P1 and P2 compared with the NoExo condition, although it was partially attained during the

421 Free trial in P1; for P3, the Pattern assistance yielded the same symmetry improvement than the

422 Free condition with respect to NoExo trial, although the Echo assistance supposed a decreased

423 in the step velocity symmetry.

424 These improvements in step velocity symmetry were because all patients increased the assisted leg's step velocity and reduced it in the non-assisted one (P1 and P2) or increased it in a lower level (P3). The origin of these velocity changes was heterogeneous across patients, and it is related to the patient's own strategies to cope with the robot's actions. For instance, P1 increased the step length and reduced step time in the paretic leg while reducing the step length of the sound leg. P2 increased the step length and time in the paretic leg while maintaining step length and increasing the step time in the sound leg. Finally, symmetry improvement in P3 was due to increasing step length and reducing step time in the paretic leg, and increasing the step length of the sound leg.

Regarding symmetry of the stance and swing phases of the gait, the assistance provided by the exoskeleton always led to a more symmetric single-support and stance step percentages compared to NoExo condition (panels 4D-4E of Figure 15); even when wearing the robot meant less symmetry than the basal condition (P1 and P2). Equally, the assistance provided by the robot improved the symmetry of swing time in all patients compared to NoExo, even when Free trials supposed a decrease of symmetry in this metric (panel 4F of Figure 15).

All patients attained the symmetry improvement in the single-support and stance phases by increasing its duration in the assisted leg and/or reducing it in the sound leg. Conversely, the strategy followed to improve symmetry in the swing time differed between subjects: P1 and P3 reduced it in both legs while P2 only increased it in the sound leg.

\section{Discussion}

This paper introduces the proof of concept of the REFLEX exoskeleton, which aims to be

445 a tool to investigate the effects of unilateral assistance in hemiparetic gait. We present two 
controllers to provide this gait assistance based on the motion of the sound leg of hemiparetic patients, looking for a natural coordination between assisted and unassisted joints. Both controllers aimed to synchronize the generated assistance with the motion of the non-paretic leg motion of the patients: the Echo strategy replicated the movement of the sound leg over the assisted one, while the Pattern strategy synchronically applied a standard healthy pattern that was adapted to the range of motion of the healthy joint. We aimed to validate the assistance provided by these controllers from two perspectives: (1) the generation of the assistive pattern, and (2) the effects of this assistance on the users' gait, focusing mainly on the compensation mechanisms developed by hemiparetic patients to attain a functional one.

\section{On the assistance generation}

The base of both assistive strategies is an AO that estimates the gait phase of the sound leg in real-time. Since this estimation is used to synchronize the assistance application, we evaluated AO's performance and its reaction to speed changes. Trials with healthy subjects at variable gait speed resulted in gait phase estimations with RMS errors lower than $2.4 \%$ of the gait phase. According to Ruiz-Garate et al., we can consider that the phase estimation is synchronized with gait if the error is lower than $10 \%$ [53]; thus, our approach is valid to coordinate the assistance provided by the prototype. Our results are in the range of those previously published by other authors, who reported RMS phase estimation errors of $3 \%$ using noncontact capacitive sensors [54], $2 \%$ using insole pressure sensors to measure the vertical ground reaction force [55], or $1.4 \%$ using an encoder to measure the hip angle [55].

Since the controllers aim to generate an assistive pattern based on the motion of the sound leg, we simulated the performance of the controllers and compared the dynamics of the assistance with the gait patterns of healthy subjects. Results pointed out that both strategies attained similarities with the master leg higher than $75 \%$. However, the average similarity on the Echo strategy (84.3\%) was significantly higher than the achieved by the Pattern strategy (77.5\%). Both strategies also were affected differently by changes in velocity. For the Echo strategy, the similarity was higher during constant speed trials (87\%) than during variable speed 
trials (82\%). In comparison, the Pattern strategy did not suppose significant differences between these trials (77\% and $78.6 \%$ during variable and constant speed trials, respectively).

Concerning the timing of the assistance, the reference pattern and the equivalent leg's motion of healthy subjects showed high synchrony. Both controllers attained a high average correlation in these movements, higher than 0.9 in both cases, although it was significantly higher for the Echo strategy $(\mathrm{R}=0.95)$ than for the Pattern strategy $(\mathrm{R}=0.9)$. Speed changes also affected these strategies differently. Echo correlation significantly decreased during variable gait speed ( $\mathrm{R}=0.94)$ compared to constant gait speed trials $(\mathrm{R}=0.98)$, while we did not identify significant changes in Pattern strategy due to speed variability.

Delays between the assistive pattern and the motion of the equivalent leg also pointed out to highly coordinated movements. Average RMS delay of the instant of maximum knee flexion remained lower than $70 \mathrm{~ms}$, which is lower than the reaction time of voluntary muscle contractions (180ms) and, therefore, it is valid for the control of robotic exoskeletons $[56,57]$.

The better performance of the Echo strategy, in terms of similarity with the master motion and correlation with the equivalent motion, was due to the controller's working principle, which directly replicated the average motion of the master leg. However, because of the five-step buffer, this controller assumed that the pattern of the master leg is repetitive between cycles. Changes in gait speed affect this repetitiveness [58], explaining the different performance between variable and constant gait speed trials. In stroke patients, this repetitiveness decreased with respect to healthy subjects [59]; thus, in these patients, the performance of this controller worsened compared with the performance in healthy subjects (see Figure 16).

\section{On the gait adaptation to assistance}

Results pointed out that healthy subjects decreased the similarity between both legs' motion because of wearing the exoskeleton's extra load. Although the robot assistance partially counteracted this effect, the device could not attain the symmetry these subjects showed during normal walking. Interestingly, the symmetry improvements yielded by the exoskeleton action 

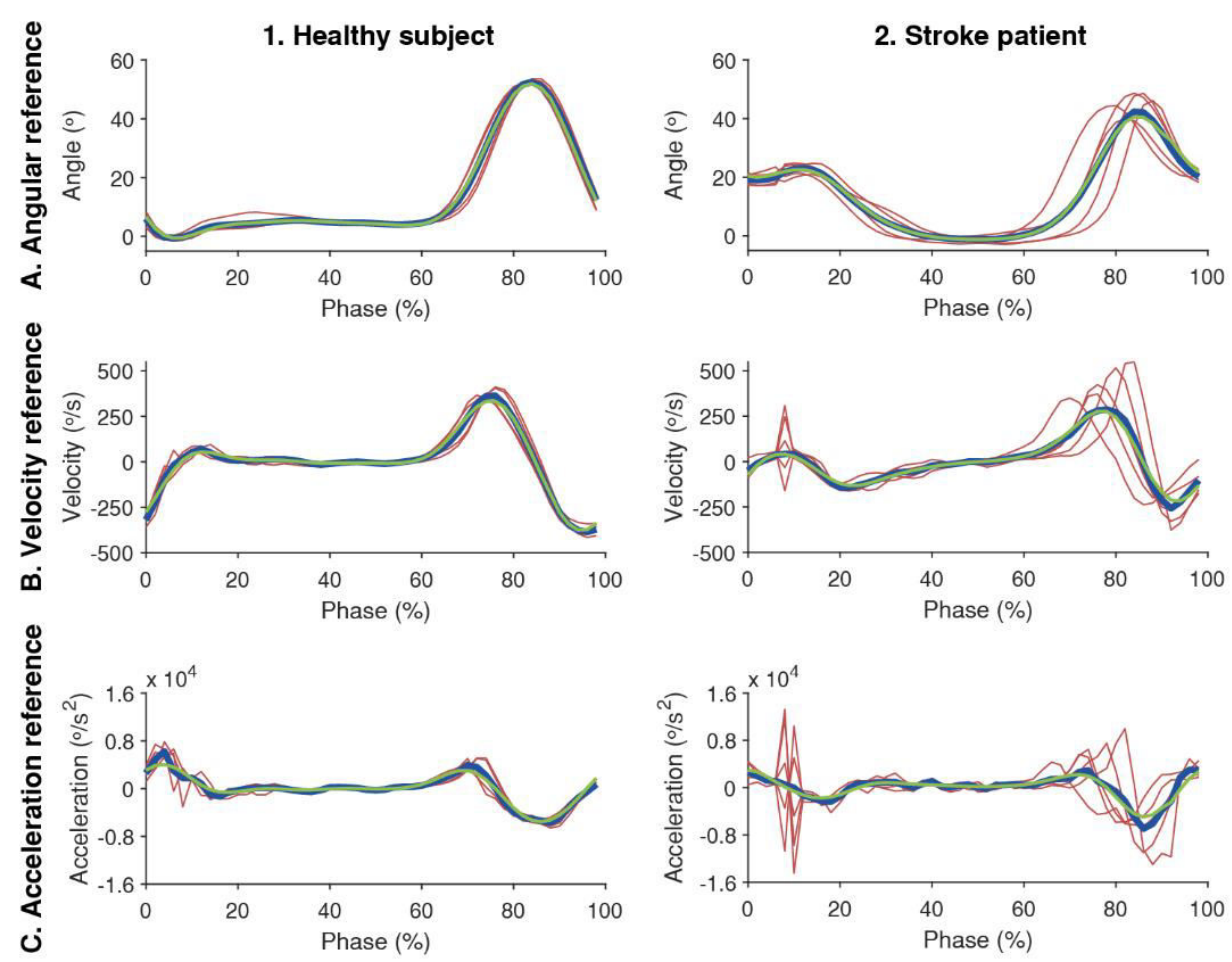

- Buffer - Average - Smoothed

Figure 16: Example of the three references generated by the Echo strategy (angle, velocity, and acceleration for rows A-C) during trials with a healthy subject (column 1) or a stroke patient (column 2). Red lines represent the content of the five steps buffer, the blue line is the average step, and the green line is the smoothed average step that serves as the pattern reference.

were not only due to changes in the assisted leg but rather the motion of the non-assisted leg also evolved to attain a higher level of symmetry. Therefore, we can conclude that healthy subjects reacted to the assistance provided by the robot by actively changing their own gait pattern to attain a more symmetric gait. This may be justified by the influence of the proprioceptive changes produced by the exoskeleton action on the gait pattern generators $[60,61]$.

The effect of the assistance over hemiparetic gait was homogeneous. All subjects increased the similarity between both limbs' motion due to the provided assistance compared with the Free condition. However, despite these improvements, the response with respect to the basal condition differed between subjects. Three out of six experimental conditions improved the symmetry with respect to the basal condition, two out of six compensated the hampering effect of wearing the robot, and only one out of six could not fully compensate it. 
512 patients. Under the Pattern strategy, two out of three patients improved the motion symmetry

513 with respect to their basal conditions, and only one subject compensated the effect of wearing

514 the robot. Conversely, under the Echo strategy, only one subject improved with respect to his

515 basal condition, one subject compensated the effect of wearing the device, and one subject could

516 not compensate for this effect. As previously mentioned, this worse performance was due to the

517 low repetitiveness of the sound limb motion that affected more to the Echo than the Pattern

518 strategy.

Compared with the Free condition, the assistance results were similar for both kinds of subjects (healthy and hemiparetic), as the assistance increased the similarity of the motion, the RoM, and the phase of maximum flexion of the knee joint. However, they differed in comparing the results with their basal condition. While the robot was not able to improve the gait symmetry in healthy subjects, it was able to assist the gait of hemiparetic subjects to achieve a more symmetric one.

The assistance provided by the robot also led to more symmetric gait features in stroke patients. The Pattern strategy improved the step length, time and velocity symmetry in all patients with respect to their basal condition, or, at least, it compensated for the hampering effect of wearing the robot. However, the Echo strategy could not achieve these improvements in one out of the three hemiparetic patients. Similarly, robotic assistance led to more symmetric single-support, stance phases, and swing time. In these cases, both assistive strategies achieved similar results. in such a way that classic compensations to attain a functional gait are decreased. In this sense, the robot's action increased the stance phase in the paretic leg and reduced the single-support phase in the non-paretic one, compensating typical disturbances in hemiparetic gait [62]. The assistance provided by the exoskeleton also yielded more symmetric step length, time, and 
velocity. These improvements could be associated with decreased asymmetric limb loading and gait inefficiency [15] and can improve patients' balance during walking [63].

Notably, these symmetry improvements are not only due to changes in the assisted limb.

On the contrary, the motion of the non-assisted leg of the patients also evolves to attain a more symmetric gait globally. Patients seemed to integrate feedback from the assisted leg to adjust the action of the non-assisted limb, improving gait performance and stability [64]. This may be explained by the action of central pattern generators located in the spinal cord using the feedback from proprioceptive muscular and tendinous receptors informing of applied loads and forces due to the exoskeleton action [61]. Although the cerebellar participation in gait pattern generation has not been clearly elucidated, it may be partly responsible for the compensation seen in our study as none of the subjects had cerebellar impairments $[65,66]$.

This assistance integration could release the non-paretic leg from excessive loading patterns that may lead to secondary musculoskeletal complications [67], since temporal asymmetry correlates with increased vertical ground reaction forces in the non-paretic leg [68]. Interestingly, these adaptations in the non-assisted leg were attained in a short time (5 minutes), and they occurred naturally without following any kind of instruction. Adaptations in the nonassisted leg were also reported by Chinimilli et al. [69], who found that unilateral knee assistance led to an increase in the stability of the unassisted leg due to inter-limb coordination in healthy subjects. For stroke patients, adaptations in the non-assisted leg were also reported during early robotic rehabilitation with the single-leg version of the HAL exoskeleton [70].

Our results on gait symmetry improvements are similar to those previously reported by other authors that aimed to assist impaired gait unilaterally. For example, Arazpour et al. described an active KAFO to improve symmetry in poliomyelitis subjects based on a FSM controller and reported symmetry improvements in swing time, stance phase percentage, and knee flexion during swing [52]. Similarly, Beyl et al. reported timing symmetry improvements in one multiple sclerosis patient when assisted by the KNEXO prototype [71]. The unilateral exoskeleton robot developed by the Shenzhen University [72] reported improvements in the 
564 joints' range of motion of three hemiparetic subjects, although their controller was based on 565 detecting the heel-strike event to trigger the assistive action. Kawamoto et al. described a 566 control strategy based on the kinematic of the non-paretic leg, similar to our Echo control, that 567 reported significant symmetry improvements in one stroke patient while HAL exoskeleton 568 assisted his gait [21]. However, in contrast with our approach, the control paradigm of these 569 examples was based on the detection of gait events, and therefore, they were not adaptive in 570 real-time to changes in gait frequency.

On the contrary, the ALEX III prototype used a pool of AOs and non-linear filters to reconstruct the joints' patterns of the non-paretic leg that acted as references for the hip and knee of the paretic limb [73]. They tested this approach in three healthy subjects simulating impairments in one leg by wearing a load in the ankle and reported gait symmetry improvements due to the action of the device. Although they reported symmetry improvements, with our approach, we assessed that the origin of these improvements is twofold, the assistance of the paretic leg and the adaptation of the non-assisted leg.

Conversely to previous studies, we compared the performance of assisting the paretic leg with the kinematic of the non-paretic leg or with a standard healthy pattern. Considering the global results with stroke patients, the Pattern strategy seemed to perform better than the Echo strategy. This suggests that providing assistance according to a normalized healthy pattern would lead to better outcomes than assisting with the motion of the non-paretic leg of the subject. As we have attained changes in the motion of both limbs, assisting the paretic limb with a healthy motion could suppose that the sound leg also would improve its motion to a healthier pattern without compensation mechanisms.

Hypothetically, this approach might lead to improvements in gait beyond compensatory strategies arisen due to hemiparesis, promoting new functional patterns with lower energetic cost. This should be explored in subsequent studies with larger sample sizes to evaluate its feasibility as an assistive strategy and its rehabilitation potential. 


\section{Conclusions}

This paper presented the REFLEX prototype and its control algorithms to evaluate the

592 effects of unilateral assistance in hemiparetic gait. REFLEX is an active KAFO that assists the knee flexion/extension of the impaired leg of stroke survivors. The control paradigm that we followed is based on the fact that the motion of both legs is delayed $180^{\circ}$, so by estimating the real-time gait phase of the non-paretic leg, we calculated the desired gait phase of the paretic one.

We also proposed two control strategies to assist the movement of the paretic limb. The Echo control strategy aimed to directly replicate the movement of the non-paretic leg, while the Adaptive healthy pattern strategy aimed to synchronically apply a standard healthy kinematic pattern to assist the movement. These two strategies promote a natural synchronization between assisted and unassisted joints. the REFLEX prototype is able to assist the gait of hemiparetic subjects coordinately with the movement of the non-paretic leg. The patients showed improved spatial and temporal symmetry due to the assistance provided by the exoskeleton. These improvements were related not only to the motion guidance imposed by the robotic exoskeleton but also to the adaptation that occurred in the non-assisted limbs, which seemed to decrease their compensatory strategies.

\section{List of abbreviations}

- AO: Adaptive Frequency Oscillator.

- Echo: Trial during which the exoskeleton assisted the user according to the Echocontrol assistive strategy.

- EMG: Electromyography.

- Free: Trial during which the subjects wore the exoskeleton, but it was mechanically decoupled from the actuator, enabling the joint's free movement.

- FSM: Finite State Machine. 
- HS: Healthy Subject.

- KAFO: Knee-Ankle-Foot orthosis.

- NoExo: Trial during which the subject was not wearing the exoskeleton.

- Pattern: Trial during which the exoskeleton assisted the user according to the Adaptive healthy pattern assistive strategy.

- RoM: Range of Motion.

- VariableSpeed: Trial during which the healthy subject walked at variable gait speed.

\section{Declarations}

\section{Ethics approval and consent to participate}

Ethical approval for the experiments was given by the Ethical Committee of the "Hospital Universitario 12 de Octubre" ( $\mathrm{N}^{\circ}$ CEIm: 20/034). All subjects gave written informed consent before participating in the study.

\section{Consent for publication}

Consent for the publication of subjects' data has been obtained for this study.

\section{Availability of data and materials}

The datasets generated and/or analyzed during the current study are available from the corresponding author on reasonable request.

\section{Competing interests}

The authors declare that they have no competing interests

\section{Funding}

This research was funded by the Spanish Ministry of Science and Innovation, project Discover2Walk (PID2019-105110RB-C31), and the Programas de Actividades I+D en la Comunidad de Madrid and Structural Funds of the EU (Robocity, S2018/NMT-4331). J.S. L-M. received a Training Program for Academic Staff fellowship (FPU16/01313) from the Ministry of Universities of the Government of Spain. 
641 We acknowledge support of the publication fee by the CSIC Open Access Publication Support

642 Initiative through its Unit of Information Resources for Research (URICI).

643 Authors' contributions

644 JSL-M and ER designed the assistive strategies; JSL-M developed and programmed the system;

645 JSL-M, JCM, and ER designed the experiments; FJS-C and JPR recruited the voluntary

646 subjects; JSL-M and FJS-C performed the experiments. All authors analyzed and interpreted the

647 data. JSL-M wrote the original document draft. All authors provided substantial feedback on

648 the manuscript. All authors read and approved the final manuscript.

\section{Acknowledgments}

650 Not applicable

\section{Bibliography} society of cardiology: Cardiovascular disease statistics 2019. Eur Heart J. 2020;41:12-85.

2. Virani SS, Alonso A, Aparicio HJ, Benjamin EJ, Bittencourt MS, Callaway CW, et al. Heart Disease and Stroke Statistics-2021 Update. Circulation [Internet]. 2021;143:E254-743. Available from: https://www.ahajournals.org/doi/10.1161/CIR.0000000000000950

3. Wafa HA, Wolfe CDA, Emmett E, Roth GA, Johnson CO, Wang Y. Burden of Stroke in Europe: Thirty-Year Projections of Incidence, Prevalence, Deaths, and Disability-Adjusted Life Years. Stroke. 2020;51:2418-27. Management of Adult Stroke Rehabilitation Care. Stroke. 2005.

5. Langhorne P, Coupar F, Pollock A. Motor recovery after stroke: a systematic review. Lancet Neurol [Internet]. Elsevier Ltd; 2009;8:741-54. Available from: http://dx.doi.org/10.1016/S1474-4422(09)70150-4 
meta-analysis. Ann Phys Rehabil Med [Internet]. Elsevier Masson SAS; 2016;59:114-24. Available from: http://dx.doi.org/10.1016/j.rehab.2016.02.001

7. Hong E. Comparison of quality of life according to community walking in stroke patients. J Phys Ther Sci [Internet]. 2015;27:2391-3. Available from: https://www.jstage.jst.go.jp/article/jpts/27/7/27_jpts-2015-177/_article

8. Vahlberg B, Cederholm T, Lindmark B, Zetterberg L, Hellström K. Factors Related to Performance-Based Mobility and Self-reported Physical Activity in Individuals 1-3 Years after Stroke: A Cross-sectional Cohort Study. J Stroke Cerebrovasc Dis [Internet]. 2013;22:e426-34. Available from: https://linkinghub.elsevier.com/retrieve/pii/S1052305713001493

9. Li S, Francisco GE, Zhou P. Post-stroke hemiplegic gait: New perspective and insights. Front Physiol. 2018;9:1-8.

10. Sheffler LR, Chae J. Hemiparetic Gait. Phys Med Rehabil Clin N Am [Internet]. Elsevier Inc; 2015;26:611-23. Available from: http://dx.doi.org/10.1016/j.pmr.2015.06.006

11. Verma R, Arya KN, Sharma P, Garg RK. Understanding gait control in post-stroke: Implications for management. J Bodyw Mov Ther [Internet]. Elsevier Ltd; 2012;16:14-21. Available from: http://dx.doi.org/10.1016/j.jbmt.2010.12.005

12. Beyaert C, Vasa R, Frykberg GE. Gait post-stroke: Pathophysiology and rehabilitation strategies. Neurophysiol Clin [Internet]. Elsevier Masson SAS; 2015;45:335-55. Available from: http://dx.doi.org/10.1016/j.neucli.2015.09.005

13. Lauzière S, Betschart M, Aissaoui R, Nadeau S. Understanding Spatial and Temporal Gait Asymmetries in Individuals Post Stroke. Int J Phys Med Rehabil [Internet]. 2014;02. Available from: https://www.omicsonline.org/open-access/understanding-spatial-and-temporalgait-asymmetries-in-individuals-post-stroke-2329-9096.1000201.php?aid=26652

14. Patterson KK, Mansfield A, Biasin L, Brunton K, Inness EL, McIlroy WE. Longitudinal Changes in Poststroke Spatiotemporal Gait Asymmetry Over Inpatient 

al. Gait Asymmetry in Community-Ambulating Stroke Survivors. Arch Phys Med Rehabil [Internet]. 2008;89:304-10.

Available

from: https://linkinghub.elsevier.com/retrieve/pii/S0003999307016498 Lower limb muscle activity underlying temporal gait asymmetry post-stroke. Clin Neurophysiol [Internet]. International Federation of Clinical Neurophysiology; 2020;131:1848-58. Available from: https://doi.org/10.1016/j.clinph.2020.04.171

17. Bohannon RW, Morton MG, Wikholm JB. Importance of four variables of walking to patients with stroke. Int J Rehabil Res [Internet]. 1991;14:246-50. Available from: http://journals.lww.com/00004356-199109000-00010

18. Li S, Chen YT, Francisco GE, Zhou P, Rymer WZ. A unifying pathophysiological account for post-stroke spasticity and disordered motor control. Front Neurol. 2019;10:1-8. Unexplored Paths and Future Directions in Gait Rehabilitation. Front Neurorobot. 2020;14. Development of an assist controller with robot suit HAL for hemiplegic patients using motion data on the unaffected side. 2014 36th Annu Int Conf IEEE Eng Med Biol Soc [Internet].

from:

712 http://ieeexplore.iee.org/document/6944273/ 
http://ieeexplore.ieee.org/document/7319468/

22. Mizukami N, Takeuchi S, Tetsuya M, Tsukahara A, Yoshida K, Matsushima A, et al. Effect of the Synchronization-Based Control of a Wearable Robot Having a Non-Exoskeletal Structure on the Hemiplegic Gait of Stroke Patients. IEEE Trans Neural Syst Rehabil Eng [Internet]. 2018;26:1011-6. Available from: https://ieeexplore.ieee.org/document/8320816/

23. Baud R, Manzoori AR, Ijspeert A, Bouri M. Review of control strategies for lowerlimb exoskeletons to assist gait. J Neuroeng Rehabil [Internet]. BioMed Central; 2021;18:119. Available from: https://doi.org/10.1186/s12984-021-00906-3

24. Yan T, Cempini M, Oddo CM, Vitiello N. Review of assistive strategies in powered lower-limb orthoses and exoskeletons. Rob Auton Syst [Internet]. Elsevier B.V.; 2015 [cited 2017 Jul 18];64:120-36. Available from: https://iopscience.iop.org/article/10.1088/1751$8113 / 44 / 8 / 085201$

25. Wong CK, Bishop L, Stein J. A wearable robotic knee orthosis for gait training. Prosthetics Orthot Int [Internet]. SAGE PublicationsSage UK: London, England; 2012 [cited 2017 Sep 29];36:113-20. Available from: http://journals.sagepub.com/doi/10.1177/0309364611428235

26. Bae J, De Rossi SMM, O’Donnell K, Hendron KL, Awad LN, Teles Dos Santos TR, et al. A soft exosuit for patients with stroke: Feasibility study with a mobile off-board actuation unit. IEEE Int Conf Rehabil Robot. 2015.

27. Awad LN, Bae J, Kudzia P, Long A, Hendron K, Holt KG, et al. Reducing Circumduction and Hip Hiking During Hemiparetic Walking Through Targeted Assistance of the Paretic Limb Using a Soft Robotic Exosuit. Am J Phys Med Rehabil. 2017;96:S157-64.

28. Srivastava S, Kao P-CC, Kim SH, Stegall P, Zanotto D, Higginson JS, et al. Assistas-Needed Robot-Aided Gait Training Improves Walking Function in Individuals Following Stroke. IEEE Trans Neural Syst Rehabil Eng. United States; 2015;23:956-63. 
742 Single-Joint Implementation of Flow Control: Knee Joint Walking Assistance for Individuals 743 with Mobility Impairment. IEEE Trans Neural Syst Rehabil Eng [Internet]. 445 HOES LANE, PISCATAWAY, NJ 08855-4141 USA: IEEE-INST ELECTRICAL ELECTRONICS ENGINEERS INC; 2020;28:934-42. Available from: https://ieeexplore.ieee.org/document/9018211/ al. Development and evaluation of a novel robotic platform for gait rehabilitation in patients with Cerebral Palsy: CPWalker. Rob Auton Syst [Internet]. Elsevier B.V.; 2017;91:101-14. Available from: http://dx.doi.org/10.1016/j.robot.2016.12.015 Hum Mov Sci. 1997;16:243-58.

32. Plotnik M, Giladi N, Hausdorff JM. A new measure for quantifying the bilateral coordination of human gait: Effects of aging and Parkinson's disease. Exp Brain Res. 2007;181:561-70. human split-belt locomotion: interaction between central mechanisms and afferent input. Exp Brain Res. Springer-Verlag; 1995.

34. Aguirre-Ollinger G, Narayan A, Yu H. Phase-Synchronized Assistive Torque Control for the Correction of Kinematic Anomalies in the Gait Cycle. IEEE Trans Neural Syst Rehabil

from: 

assist drop-foot gait. IEEE Trans NEURAL Syst Rehabil Eng [Internet]. 2004;12:24-31. Available from: http://dx.doi.org/10.1109/TNSRE.2003.823266 force condition for HAL. Adv Robot. 2005;19:717-34. in-the-loop optimization of exoskeleton assistance during walking. Science (80- ) [Internet].

from:

https://www.sciencemag.org/lookup/doi/10.1126/science.aal5054

39. Awad LN, Bae J, Kathleen OD, Rossi SMM De, Hendron K, Sloot LH, et al. A soft robotic exosuit improves walking in patients after stroke. Sci Transl Med [Internet]. 2017;9:eaai9084. Available from: https://www.scopus.com/inward/record.uri?eid=2-s2.085026198983\&doi=10.1126\%2Fscitranslmed.aai9084\&partnerID=40\&md5=204eb4d534ea98b bb6aac4132cbdd840 Voluntary motion support control of Robot Suit HAL triggered by bioelectrical signal for hemiplegia. 2010 Annu Int Conf IEEE Eng Med Biol Soc EMBC'10. 2010;462-6.

41. Gordon KE, Ferris DP. Learning to walk with a robotic ankle exoskeleton. J Biomech

from: http://linkinghub.elsevier.com/retrieve/pii/S0021929006004866 early after stroke with a new exoskeleton - The hybrid assistive limb: A study of safety and feasibility. J Neuroeng Rehabil [Internet]. BioMed Central; 2014 [cited 2018 Jun 13];11:92. 
43. Fleischer $\mathrm{C}$, Hommel Gü. A Human-Exoskeleton Interface Utilizing

793 Electromyography. IEEE Trans Robot [Internet]. 2008 [cited 2018 Nov 30];24:872-82.

794 Available from: http://ieeexplore.ieee.org/document/4560058/

44. Ronsse R, De Rossi SMM, Vitiello N, Lenzi T, Carrozza MC, Ijspeert AJ. Real-Time

Estimate of Velocity and Acceleration of Quasi-Periodic Signals Using Adaptive Oscillators.

797

IEEE Trans Robot [Internet]. 2013;29:783-91. Available from:

http://ieeexplore.ieee.org/document/6428719/

45. van Dijk W, Meijneke C, van der Kooij H. Evaluation of the Achilles Ankle

Exoskeleton. IEEE Trans Neural Syst Rehabil Eng [Internet]. 2017;25:151-60. Available from:

801

http://ieeexplore.ieee.org/document/7404039/

46. Wang WJ, Li J, Li W Da, Sun LN. An Echo-Based Gait Phase Determination Method of Lower Limb Prosthesis. Adv Mater Res [Internet]. Trans Tech Publications; 2013 [cited 2017

47. Koopman B, van Asseldonk EHF, van der Kooij H. Speed-dependent reference joint trajectory generation for robotic gait support. J Biomech [Internet]. Elsevier; 2014;47:1447-58.

Available from: http://dx.doi.org/10.1016/j.jbiomech.2014.01.037

808

48. Hogan N. The mechanics of multi-joint posture and movement control. Biol Cybern

[Internet]. 1985 [cited 2017 Apr 24];52:315-31. Available from: http://www.ncbi.nlm.nih.gov/pubmed/4052499

49. Wehbi F el zahraa, Huo W, Amirat Y, Rafei M El, Khalil M, Mohammed S. Active impedance control of a knee-joint orthosis during swing phase. 2017 Int Conf Rehabil Robot

813 [Internet]. IEEE; 2017. p. 435-40. Available from:

814 https://ieeexplore.ieee.org/document/8009286/ 
817 2015;12:112. Available from: https://www.scopus.com/inward/record.uri?eid=2-s2.084994508627\&doi=10.5772\%2F60782\&partnerID=40\&md5=58ef4ba915eba6091871198705f1 $9 \mathrm{~d} 51$

51. Hurmuzlu Y, Basdogan C, Carollo JJ. Presenting joint kinematics of human locomotion using phase plane portraits and Poincaré maps. J Biomech [Internet]. 1994;27:1495al. Evaluation of gait symmetry in poliomyelitis subjects: Comparison of a conventional kneeankle-foot orthosis and a new powered knee-ankle-foot orthosis. Prosthet Orthot Int [Internet]. 2016;40:689-95. Available from: https://www.scopus.com/inward/record.uri?eid=2-s2.084995878373\&doi=10.1177\%2F0309364615596063\&partnerID=40\&md5=b5eb904a7748e9f7 6f3381d2fab0cfec

53. Ruiz Garate V, Parri A, Yan T, Munih M, Molino Lova R, Vitiello N, et al.

830 Experimental Validation of Motor Primitive-Based Control for Leg Exoskeletons during 831 Continuous Multi-Locomotion Tasks. Front Neurorobot [Internet]. 2017;11. Available from: http://journal.frontiersin.org/article/10.3389/fnbot.2017.00015/full on noncontact capacitive sensing and adaptive oscillators. IEEE Trans Biomed Eng. 2017;64:2419-30. smooth real-time estimate of gait phase for wearable robotics. Auton Robots. Springer US; $2017 ; 41: 759-74$. 
Sensitive Insoles for Real-Time Gait-Related Applications. Sensors [Internet]. 2020;20:1448.

Available from: https://www.mdpi.com/1424-8220/20/5/1448

58. Fukuchi CA, Fukuchi RK, Duarte M. Effects of walking speed on gait biomechanics in healthy participants: A systematic review and meta-analysis. Syst Rev. Systematic Reviews; 2019;8:1-11.

59. Kao P-C, Dingwell JB, Higginson JS, Binder-Macleod S. Dynamic instability during post-stroke hemiparetic walking. Gait Posture [Internet]. 2014;40:457-63. Available from: https://linkinghub.elsevier.com/retrieve/pii/S0966636214005463

60. He B, Lu Q, Wang Z. Coupling effect analysis between the central nervous system and the CPG network with proprioception. Robotica [Internet]. 2015;33:1281-94. Available from: https://www.cambridge.org/core/product/identifier/S0263574714000708/type/journal_article Human Gaits in OpenSim Simulator. 2019 Int Jt Conf Neural Networks [Internet]. IEEE; 2019. p. 1-8. Available from: https://ieeexplore.ieee.org/document/8852163/ American Academy of Physical Medicine and Rehabilitation; 2014;6:635-42. Available from: http://dx.doi.org/10.1016/j.pmrj.2013.12.017 spatiotemporal gait asymmetry and balance in individuals with chronic stroke. J Appl Biomech. 2014;30:31-6. 
and Gait Performance. J Mot Behav [Internet]. 2015;47:563-74. Available from:

65. Guertin PA. Central Pattern Generator for Locomotion: Anatomical, Physiological, and Pathophysiological Considerations. Front Neurol [Internet]. 2013;3:1-15. Available from: http://journal.frontiersin.org/article/10.3389/fneur.2012.00183/abstract 2015;114:1693-704. loading patterns of the non-paretic and paretic legs during post-stroke gait. Gait Posture http://dx.doi.org/10.1016/j.gaitpost.2016.07.019

68. Kim CM, Eng JJ. Symmetry in vertical ground reaction force is accompanied by symmetry in temporal but not distance variables of gait in persons with stroke. Gait Posture. $2003 ; 18: 23-8$.

69. Chinimilli PT, Rezayat Sorkhabadi SM, Zhang W. Assessment of Human Dynamic

[Internet]. 445 HOES LANE, PISCATAWAY, NJ 08855-4141 USA: IEEE-INST ELECTRICAL ELECTRONICS ENGINEERS INC; 2020;28:669-78. Available from: https://ieeexplore.ieee.org/document/8974246/ of bilateral gait coordination in hemiparetic stroke patients after early robotic intervention. Front Neurosci. 2018;12:1-11. 
893 Rehabilitation of Gait. Adv Robot [Internet]. Taylor \& Francis Group; 2011 [cited 2020 May 894 5];25:513-35. Available from:

895 https://www.tandfonline.com/doi/full/10.1163/016918611X558225

896 72. Wei D, Li Z, Wei Q, Su H, Song BB, He W, et al. Human-in-the-Loop Control 897 Strategy of Unilateral Exoskeleton Robots for Gait Rehabilitation. IEEE Trans Cogn Dev Syst 898 [Internet]. 2019;13:1-1. Available from: https://ieeexplore.ieee.org/document/8906035/ 900 Gait Symmetry in Robot-Assisted Gait Training. 2014 IEEE Int Conf Robot Autom. 2014. p. $901 \quad 724-9$.

902 